\title{
Supervised learning in adaptive DNA strand displacement networks (SUPPORTING INFORMATION)
}

\author{
Matthew R. Lakin*1,2,3 and Darko Stefanovic ${ }^{2,3}$ \\ ${ }^{1}$ Dept. of Chemical \& Biological Engineering, University of New Mexico, Albuquerque, NM, USA \\ ${ }^{2}$ Dept. of Computer Science, University of New Mexico, Albuquerque, NM, USA \\ ${ }^{3}$ Center for Biomedical Engineering, University of New Mexico, Albuquerque, NM, USA
}

\section{List of Supporting Figures}

Figure S1 Learning performance distribution, for a range of starting points, after 15 training rounds . . . . . . 3

Figure S2 Learning performance distribution over time, from 2-valued starting points, up to 15 training rounds . 4

Figure S3 Learning performance distribution over time, from 4-valued starting points, up to 15 training rounds . 5

Figure S4 Learning performance distribution over time, from 6-valued starting points, up to 15 training rounds . 6

Figure S5 Learning performance distribution over time, from 8-valued starting points, up to 15 training rounds . 7

Figure S6 Learning performance distribution over time, from 10-valued starting points, up to 15 training rounds . 8

Figure S7 Learning performance distribution over time, from 0-valued starting point, up to 15 training rounds . . 9

Figure S8 Histograms of final weight RMSE values in simulations with noise, matching $y$-axes . . . . . . . . 10

Figure S9 Histograms of final weight RMSE values in simulations with noise, independent $y$-axes . . . . . . . . 11

Figure S10 Learning performance distribution over time, $\widehat{w_{1}}=-2, \widehat{w_{2}}=-2$, up to 20 training rounds . . . . . . 13

Figure S11 Learning performance distribution over time, $\widehat{w_{1}}=-2, \widehat{w_{2}}=2$, up to 20 training rounds . . . . . . . 13

Figure S12 Learning performance distribution over time, $\widehat{w_{1}}=2, \widehat{w_{2}}=2$, up to 20 training rounds . . . . . . . . . 14

Figure S13 Learning performance distribution over time, $\widehat{w_{1}}=2, \widehat{w_{2}}=-2$, up to 20 training rounds . . . . . . . . 14

Figure S14 Learning performance distribution over time, $\widehat{w_{1}}=-4, \widehat{w_{2}}=-4$, up to 20 training rounds . . . . . . . 15

Figure S15 Learning performance distribution over time, $\widehat{w_{1}}=-4, \widehat{w_{2}}=4$, up to 20 training rounds . . . . . . . 15

Figure S16 Learning performance distribution over time, $\widehat{w_{1}}=4, \widehat{w_{2}}=4$, up to 20 training rounds . . . . . . . . 16

Figure S17 Learning performance distribution over time, $\widehat{w_{1}}=4, \widehat{w_{2}}=-4$, up to 20 training rounds . . . . . . . 16

Figure S18 Learning performance distribution over time, $\widehat{w_{1}}=-6, \widehat{w_{2}}=-6$, up to 20 training rounds . . . . . . . 17

Figure S19 Learning performance distribution over time, $\widehat{w_{1}}=-6, \widehat{w_{2}}=6$, up to 20 training rounds . . . . . . . . 17

Figure S20 Learning performance distribution over time, $\widehat{w_{1}}=6, \widehat{w_{2}}=6$, up to 20 training rounds . . . . . . . 18

Figure S21 Learning performance distribution over time, $\widehat{w_{1}}=6, \widehat{w_{2}}=-6$, up to 20 training rounds . . . . . . . 18

Figure S22 Learning performance distribution over time, $\widehat{w_{1}}=-8, \widehat{w_{2}}=-8$, up to 20 training rounds . . . . . . . 19

Figure S23 Learning performance distribution over time, $\widehat{w_{1}}=-8, \widehat{w_{2}}=8$, up to 20 training rounds . . . . . . . 19

Figure S24 Learning performance distribution over time, $\widehat{w_{1}}=8, \widehat{w_{2}}=8$, up to 20 training rounds . . . . . . . . 20

Figure S25 Learning performance distribution over time, $\widehat{w_{1}}=8, \widehat{w_{2}}=-8$, up to 20 training rounds . . . . . . . 20

Figure S26 Learning performance distribution over time, $\widehat{w_{1}}=-10, \widehat{w_{2}}=-10$, up to 20 training rounds . . . . 21

Figure S27 Learning performance distribution over time, $\widehat{w_{1}}=-10, \widehat{w_{2}}=10$, up to 20 training rounds . . . . . 21

Figure S28 Learning performance distribution over time, $\widehat{w_{1}}=10, \widehat{w_{2}}=10$, up to 20 training rounds . . . . . . 22

Figure S29 Learning performance distribution over time, $\widehat{w_{1}}=10, \widehat{w_{2}}=-10$, up to 20 training rounds . . . . . 22

Figure S30 Learning performance distribution over time, $\widehat{w_{1}}=0, \widehat{w_{2}}=0$, up to 20 training rounds . . . . . . . 23

${ }^{*}$ Corresponding author (mlakin@cs.unm.edu) 


\section{S1 Additional Results From Weight Space Scanning Simulations}

This section presents additional results from weight space scanning simulations, extending those presented in the main text. For the initial weight space scanning experiments, target weight values were chosen from the interval $[-10,10]$ at regular intervals of 1 unit and initial weight values were chosen on the diagonals of the weight space. These results are presented in Figure S1, and show that the learning circuit can achieve a uniform, low weight distribution of RMSE values irrespective of the starting weight values. This indicates that the system can learn different weight values without inherent bias. Additional results showing the weight RMSE values across the weight space at different points in the training schedule, which show how the learning proceeds over time, are presented in Figures S2-S7. These plots show how the distribution of weight RMSE values across the weight space evolves toward the low and uniform weight RMSE distribution that was observed after the 15round training schedules were completed. The occurrence of isolated points with higher RMSE in the plots after 15 training rounds may be attributed to buffer exhaustion, which is discussed further in the main text, and below. 

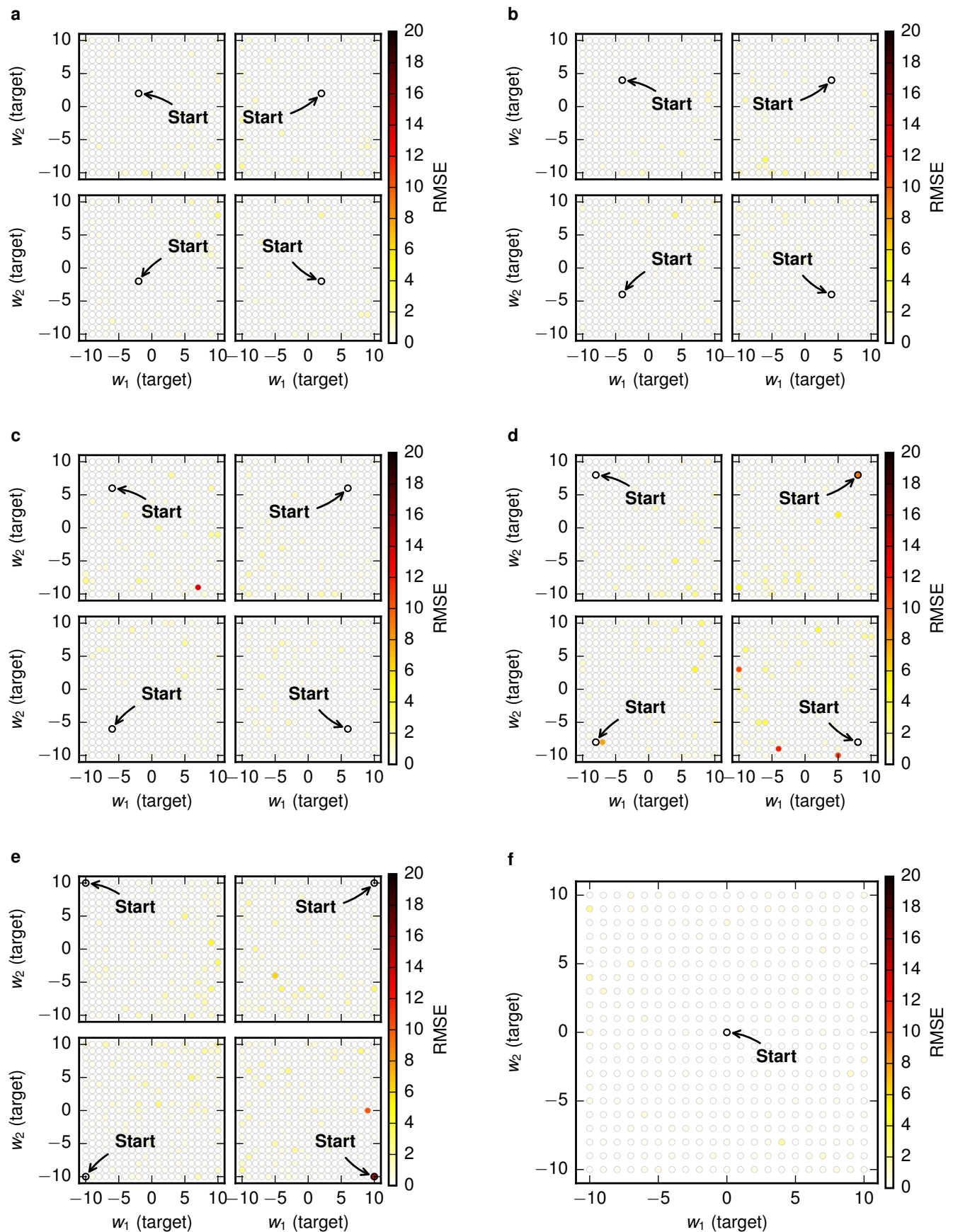

Figure S1: Scatter plots showing the distribution of learning performance across a range of target weight values for several different initial weight values, extending the data presented in the main text. The position of each marker represents a pair of weight values in the two-dimensional weight space, and each marker is color-coded to represent the final RMSE in the weight approximations when attempting to learn those weight values from the weight values marked "Start." In each case, the average RMSE in the weight approximations was computed after a 15-round training schedule using input values for each training instance drawn from a uniform distribution over $[-10,10]$. We observe a low and uniform distribution of final RMSE values, indicating that the system can learn a range of different weight values without inherent bias. The occurrence of isolated points with higher RMSE may be attributed to buffer exhaustion, which is discussed in the main text and in the Supporting Information. 

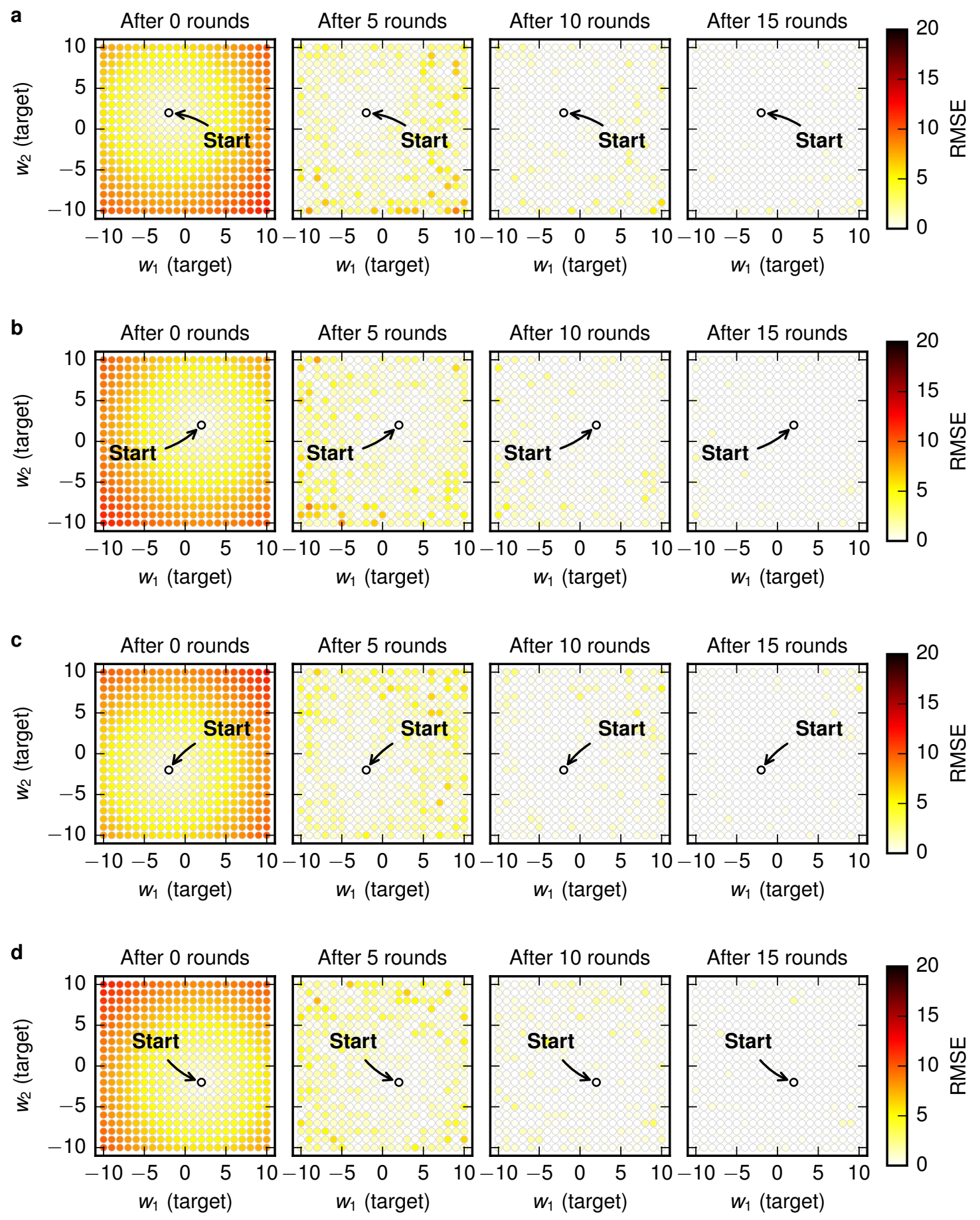

Figure S2: Scatter plots showing the learning performance over time for a range of target weight values and several initial weight values such that, initially, $\widehat{w_{1}}$ and $\widehat{w_{2}}$ both have magnitude 2 . The position of each marker represents a pair of weight values in the two-dimensional weight space, and each marker is color-coded to represent the final RMSE in the weight approximations when attempting to learn those weight values from the weight values marked "Start," after 0, 5, 10, and 15 training rounds. The input values for each training instance drawn from a uniform distribution over $[-10,10]$. In the initial pane, the weight RMSE is simply a function of distance from the starting weight approximation in the two-dimensional weight space, but this evolves toward a low and uniform distribution of weight RMSE values after 15 training rounds, indicating that the system can learn a range of different weight values without inherent bias. The occurrence of isolated points with higher RMSE may be attributed to buffer exhaustion, which is discussed in the main text and in the Supporting Information. 

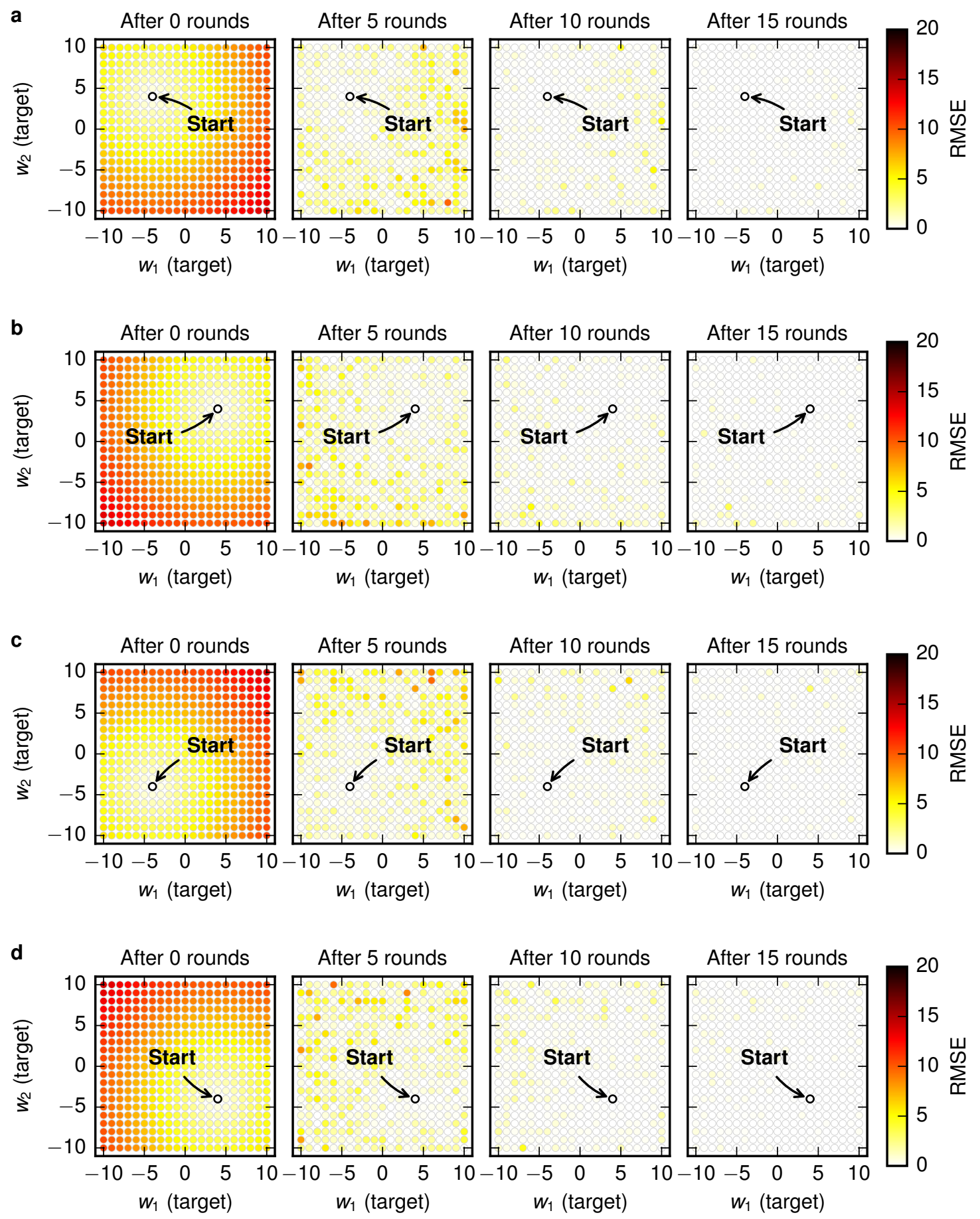

Figure S3: Scatter plots showing the learning performance over time for a range of target weight values and several initial weight values such that, initially, $\widehat{w_{1}}$ and $\widehat{w_{2}}$ both have magnitude 4 . The position of each marker represents a pair of weight values in the two-dimensional weight space, and each marker is color-coded to represent the final RMSE in the weight approximations when attempting to learn those weight values from the weight values marked "Start," after 0, 5, 10, and 15 training rounds. The input values for each training instance drawn from a uniform distribution over $[-10,10]$. In the initial pane, the weight RMSE is simply a function of distance from the starting weight approximation in the two-dimensional weight space, but this evolves toward a low and uniform distribution of weight RMSE values after 15 training rounds, indicating that the system can learn a range of different weight values without inherent bias. The occurrence of isolated points with higher RMSE may be attributed to buffer exhaustion, which is discussed in the main text and in the Supporting Information. 

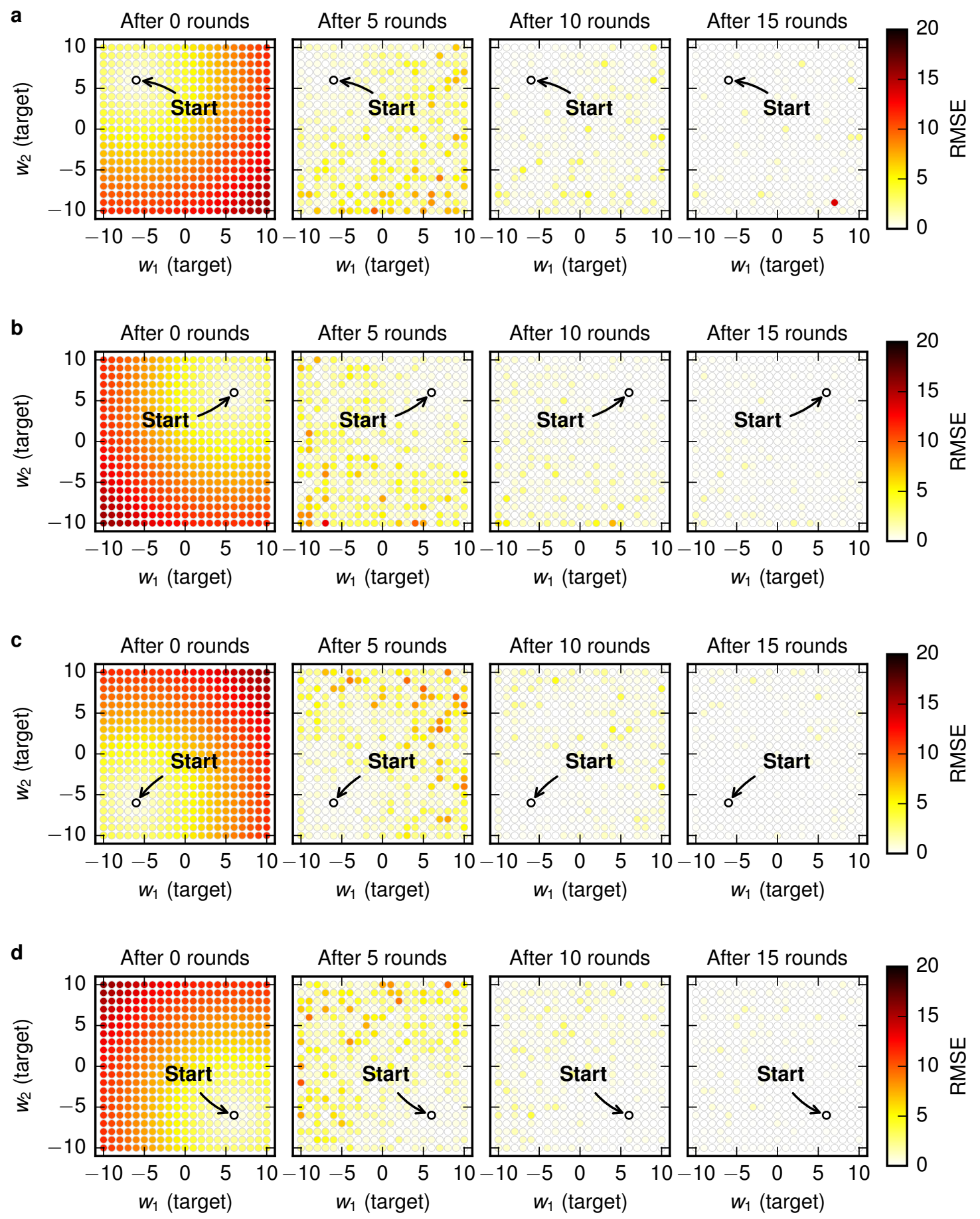

Figure S4: Scatter plots showing the learning performance over time for a range of target weight values and several initial weight values such that, initially, $\widehat{w_{1}}$ and $\widehat{w_{2}}$ both have magnitude 6 . The position of each marker represents a pair of weight values in the two-dimensional weight space, and each marker is color-coded to represent the final RMSE in the weight approximations when attempting to learn those weight values from the weight values marked "Start," after 0, 5, 10, and 15 training rounds. The input values for each training instance drawn from a uniform distribution over $[-10,10]$. In the initial pane, the weight RMSE is simply a function of distance from the starting weight approximation in the two-dimensional weight space, but this evolves toward a low and uniform distribution of weight RMSE values after 15 training rounds, indicating that the system can learn a range of different weight values without inherent bias. The occurrence of isolated points with higher RMSE may be attributed to buffer exhaustion, which is discussed in the main text and in the Supporting Information. 

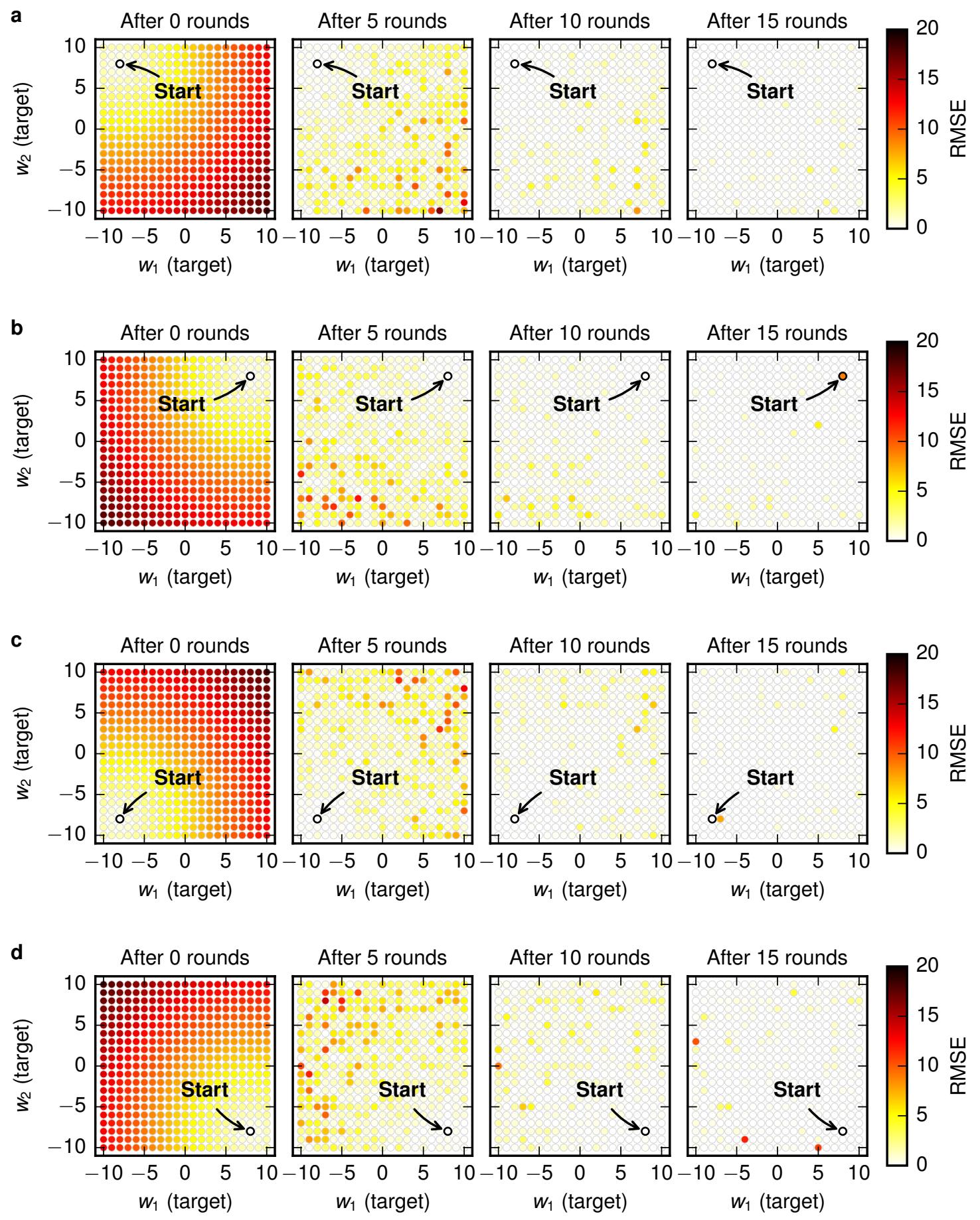

Figure S5: Scatter plots showing the learning performance over time for a range of target weight values and several initial weight values such that, initially, $\widehat{w_{1}}$ and $\widehat{w_{2}}$ both have magnitude 8 . The position of each marker represents a pair of weight values in the two-dimensional weight space, and each marker is color-coded to represent the final RMSE in the weight approximations when attempting to learn those weight values from the weight values marked "Start," after 0, 5, 10, and 15 training rounds. The input values for each training instance drawn from a uniform distribution over $[-10,10]$. In the initial pane, the weight RMSE is simply a function of distance from the starting weight approximation in the two-dimensional weight space, but this evolves toward a low and uniform distribution of weight RMSE values after 15 training rounds, indicating that the system can learn a range of different weight values without inherent bias. The occurrence of isolated points with higher RMSE may be attributed to buffer exhaustion, which is discussed in the main text and in the Supporting Information. 



Figure S6: Scatter plots showing the learning performance over time for a range of target weight values and several initial weight values such that, initially, $\widehat{w_{1}}$ and $\widehat{w_{2}}$ both have magnitude 10 . The position of each marker represents a pair of weight values in the two-dimensional weight space, and each marker is color-coded to represent the final RMSE in the weight approximations when attempting to learn those weight values from the weight values marked "Start," after 0, 5, 10, and 15 training rounds. The input values for each training instance drawn from a uniform distribution over $[-10,10]$. In the initial pane, the weight RMSE is simply a function of distance from the starting weight approximation in the two-dimensional weight space, but this evolves toward a low and uniform distribution of weight RMSE values after 15 training rounds, indicating that the system can learn a range of different weight values without inherent bias. The occurrence of isolated points with higher RMSE may be attributed to buffer exhaustion, which is discussed in the main text and in the Supporting Information. 


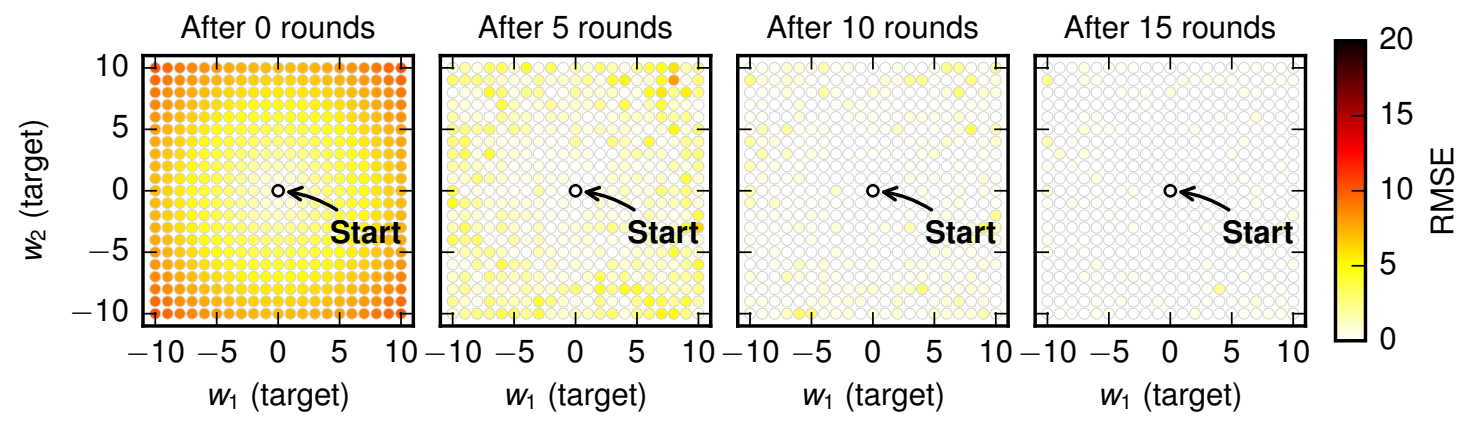

Figure S7: Scatter plots showing the learning performance over time for a range of target weight values and initial weight approximations $\widehat{w_{1}}=0$ and $\widehat{w_{2}}=0$. The position of each marker represents a pair of weight values in the two-dimensional weight space, and each marker is color-coded to represent the final RMSE in the weight approximations when attempting to learn those weight values from the weight values marked "Start," after 0, 5, 10, and 15 training rounds. The input values for each training instance drawn from a uniform distribution over $[-10,10]$. In the initial pane, the weight RMSE is simply a function of distance from the starting weight approximation in the two-dimensional weight space, but this evolves toward a low and uniform distribution of weight RMSE values after 15 training rounds, indicating that the system can learn a range of different weight values without inherent bias. The occurrence of isolated points with higher RMSE may be attributed to buffer exhaustion, which is discussed in the main text and in the Supporting Information.

\section{S2 Weight RMSE Histograms From Noise Simulations}

In this section, we present additional results from the learning simulations with noise. Figure S8 presents histograms showing the distributions of the final weight RMSE values after 15-round training schedules carried out with different amounts of noise, that is, for normally-distributed noise with mean 0 and standard deviation $\sigma$. We used values of $\sigma$ ranging from 0 to 5 . The same data are replotted in Figure S9 with independent $y$-axes for each plot, so the shapes of the distributions for larger values of $\sigma$ are more visible. These plots show that it is possible to achieve low final RMSE values with large amounts of noise, but the distributions of the final weight RMSE values are much broader and long-tailed, with some simulations leading to large amounts of error after the training completes. The plot for $\sigma=0$ also shows the tight distribution of weight RMSE values near to zero in the absence of noise, which illustrates the reliability of our learning circuit design when there is no noise in the training inputs. 


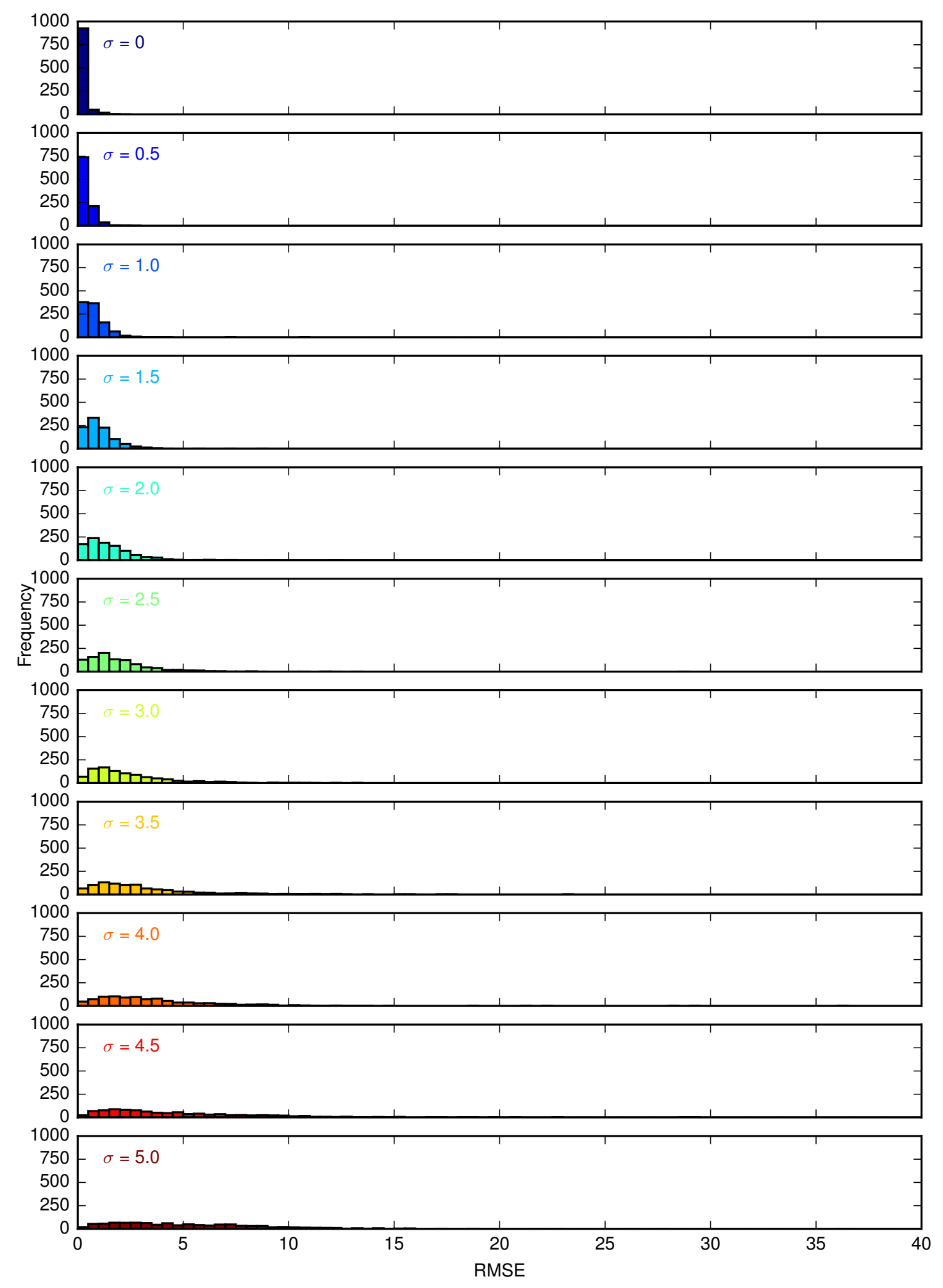

Figure S8: Histograms of final weight RMSE values for learning simulations with different amounts of noise (different standard deviations $\sigma$ of the noise distribution). The RMSE values of the weight approximations compared to the target weight values were computed after completing 1,000 training schedules, each 15 rounds in length. The data show how the weight RMSE distribution widens, with more high-RMSE results in the simulations with higher $\sigma$ values. 


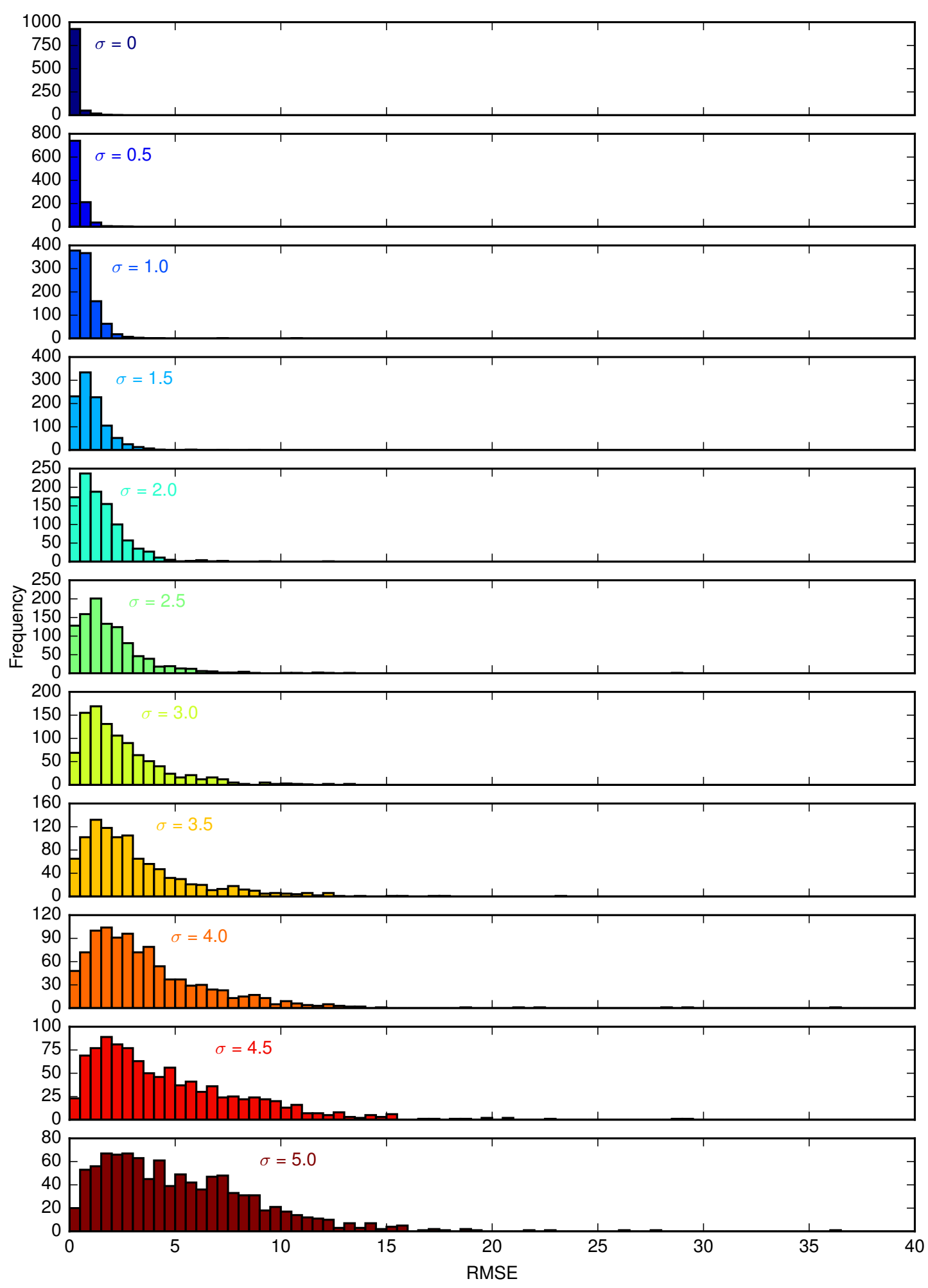

Figure S9: Histograms of final weight RMSE values for learning simulations with different amounts of noise (different standard deviations $\sigma$ of the noise distribution), replotted with independent $y$-axes for each plot. The RMSE values of the weight approximations compared to the target weight values were computed after completing 1,000 training schedules, each 15 rounds in length. The data show how the weight RMSE distribution widens, with more high-RMSE results in the simulations with higher $\sigma$ values. 


\section{S3 Results From Extended Weight Space Scanning Simulations With and With- out Buffer Replenishment}

To investigate the effects of buffer exhaustion on the results of running learning simulations, we re-ran the previous set of weight space scanning experiments, but extended the lengths of the training schedules from 15 rounds to 20 rounds. The addition training input values were chosen from a uniform distribution over $[-10,10]$, as before. The results from these simulations were plotted as above and are presented in Figures S10-S30 for various initial weight values. In all of these figures, the position of each marker represents a pair of weight values in the two-dimensional weight space, and each marker is color-coded to represent the final RMSE in the weight approximations when attempting to learn those weight values from the weight values marked "Start," after 0, 5, 10, 15, and 20 training rounds. The input values for each training instance were drawn from a uniform distribution over $[-10,10]$. In the (a) subfigures there was no buffer replenishment, whereas in the (b) subfigures the initial quantity of each buffered species is added every 5 training rounds. These plots show that the weight RMSE values across the weight space decrease to a minimum value that is close to zero after around 15 training rounds, and then begins to increase again. Further examination of the species timecourses showed that this effect is due to buffer exhaustion: as the buffers of inactive strand displacement gates run out, the circuit cannot operate correctly any more. This causes the species added in subsequent training rounds to build up, leading to observed increases in weight RMSE values. If the quantities of inactive buffered species are periodically replenished (subfigures (b) of Figures S10-S30) the increase in weight RMSE values after 15 training rounds is significantly reduced compared to when the buffer is not replenished (subfigures (a) of Figures S10-S30). Furthermore, prior to 15 training rounds the results from both plots are identical. These data show that replenishing the buffered species can extend the useful life of the learning circuit without otherwise affecting its operation. 

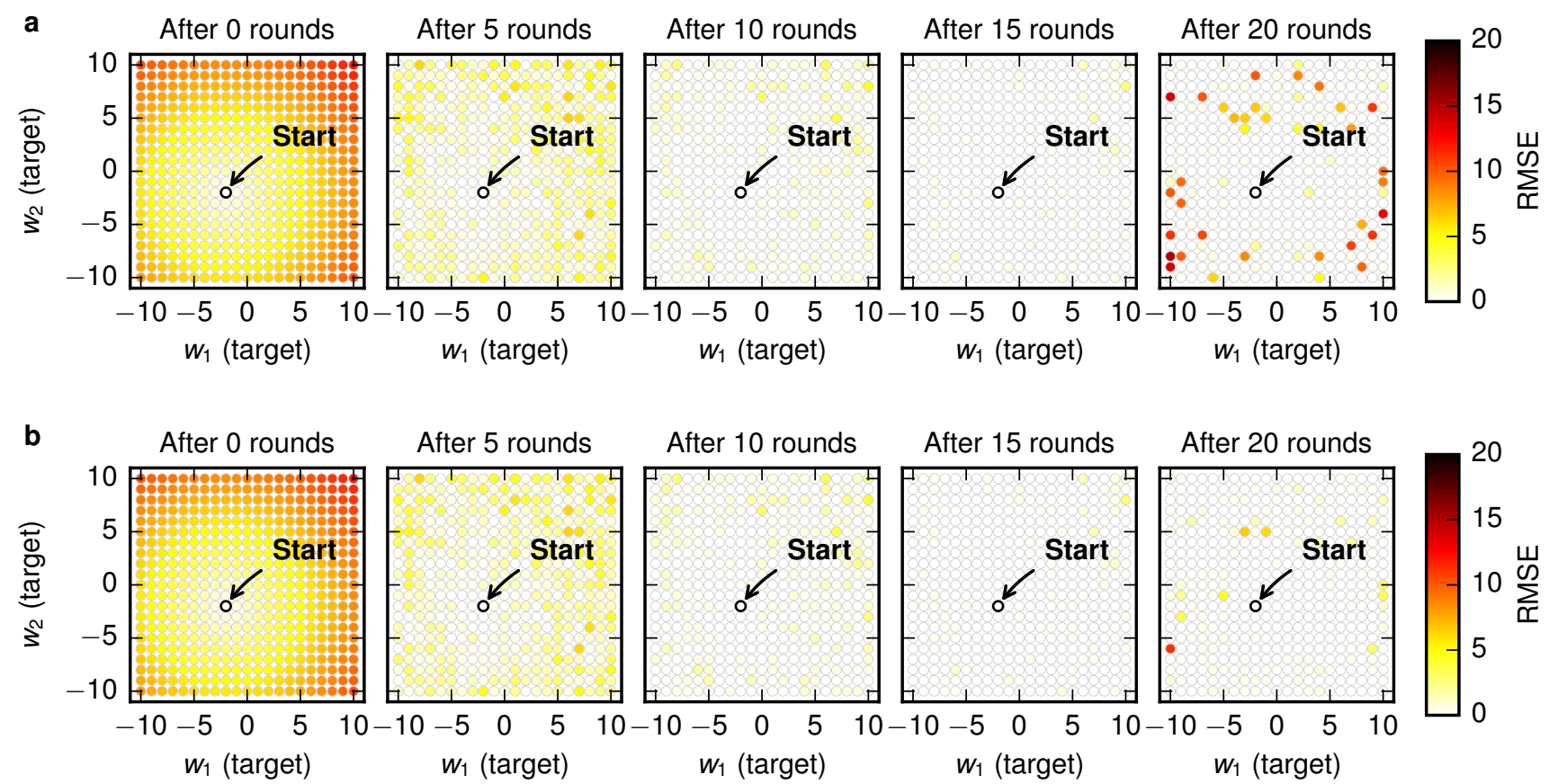

Figure S10: Scatter plots showing the learning performance over time for initial weight values $\widehat{w_{1}}=-2$ and $\widehat{w_{2}}=-2$. (a) No buffer replenishment. (b) Buffer replenished every 5 rounds.
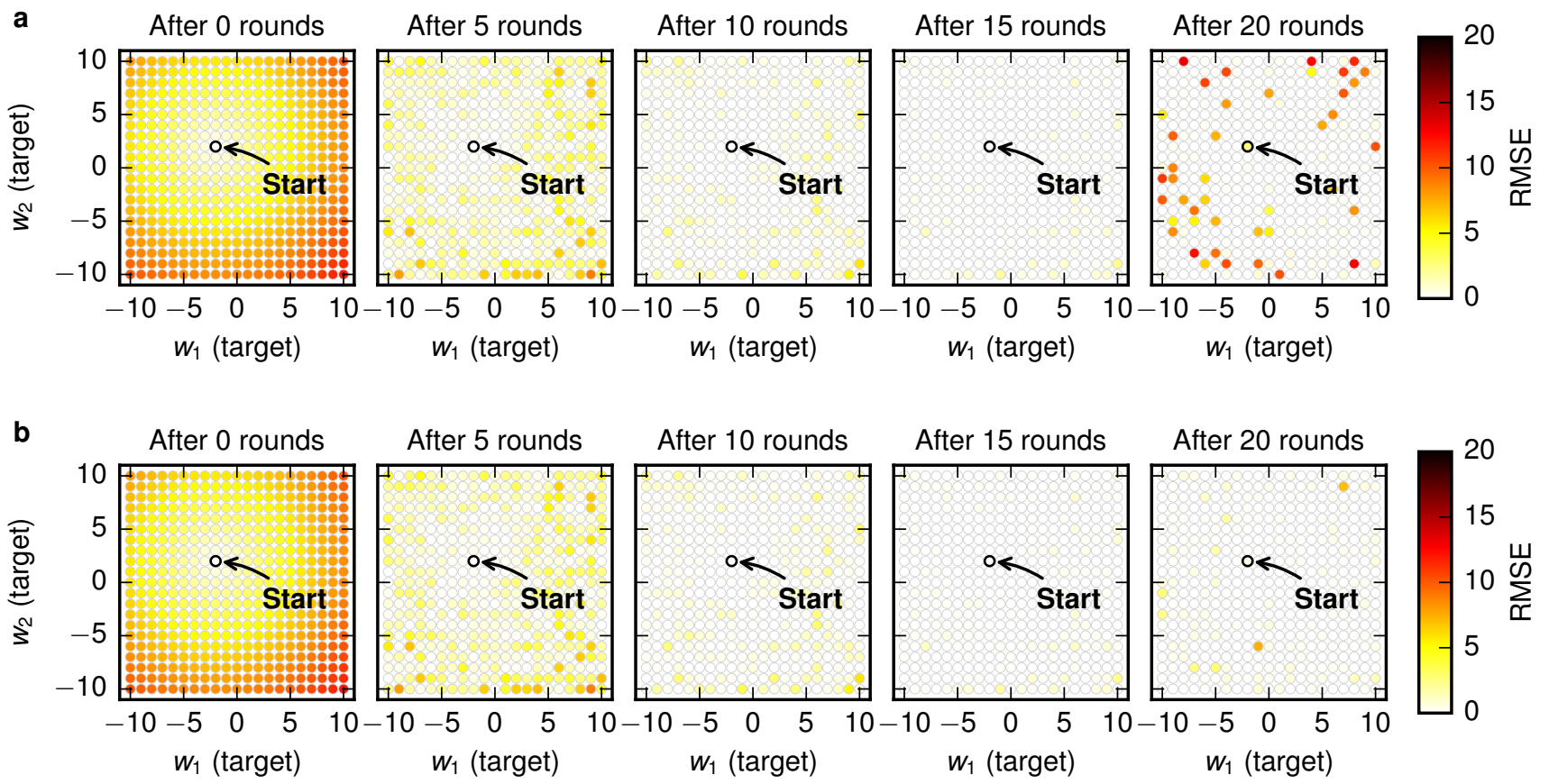

Figure S11: Scatter plots showing the learning performance over time for initial weight values $\widehat{w_{1}}=-2$ and $\widehat{w_{2}}=2$. (a) No buffer replenishment. (b) Buffer replenished every 5 rounds. 

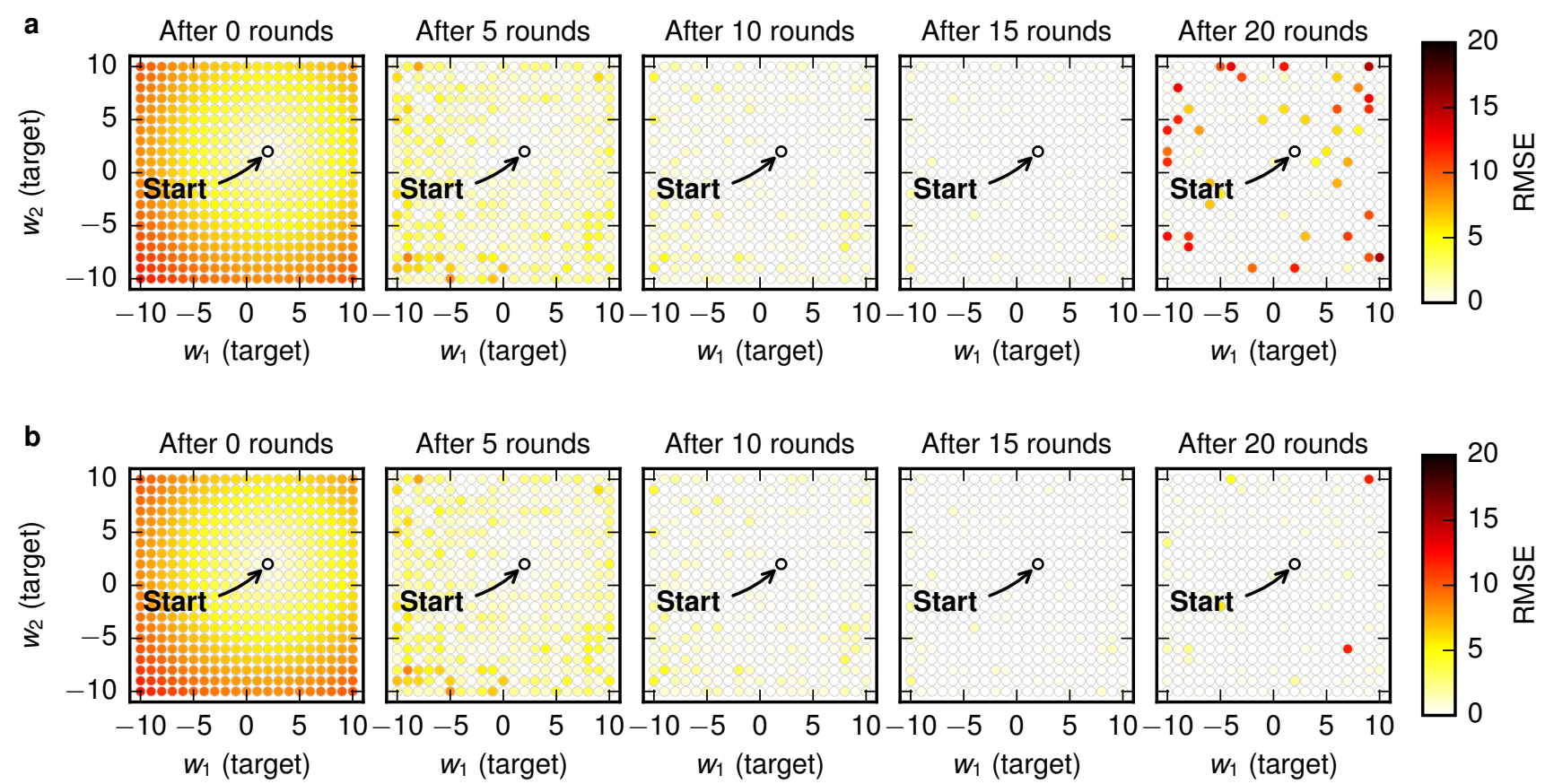

Figure S12: Scatter plots showing the learning performance over time for initial weight values $\widehat{w_{1}}=2$ and $\widehat{w_{2}}=2$. (a) No buffer replenishment. (b) Buffer replenished every 5 rounds.
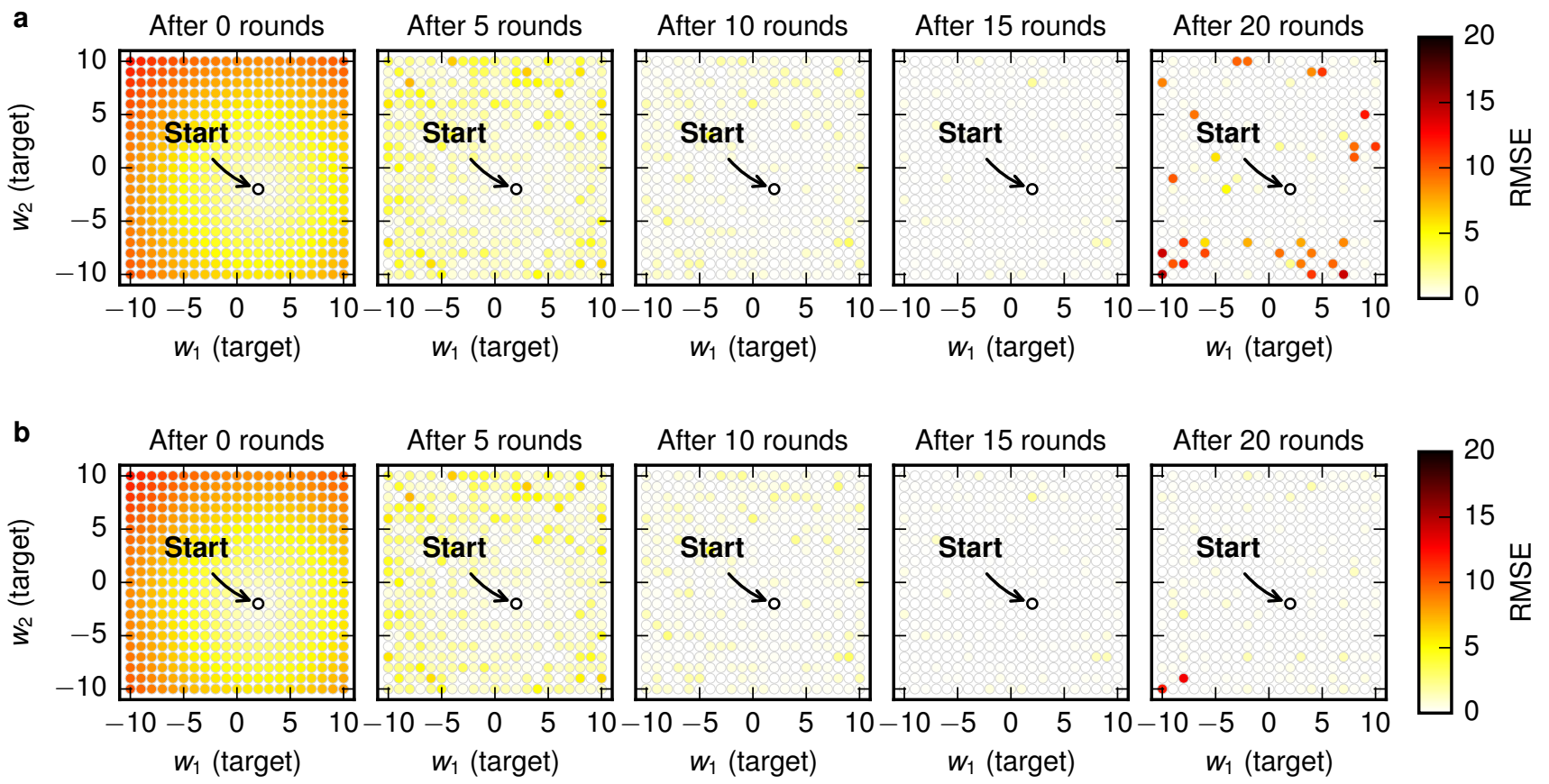

Figure S13: Scatter plots showing the learning performance over time for initial weight values $\widehat{w_{1}}=2$ and $\widehat{w_{2}}=-2$. (a) No buffer replenishment. (b) Buffer replenished every 5 rounds. 

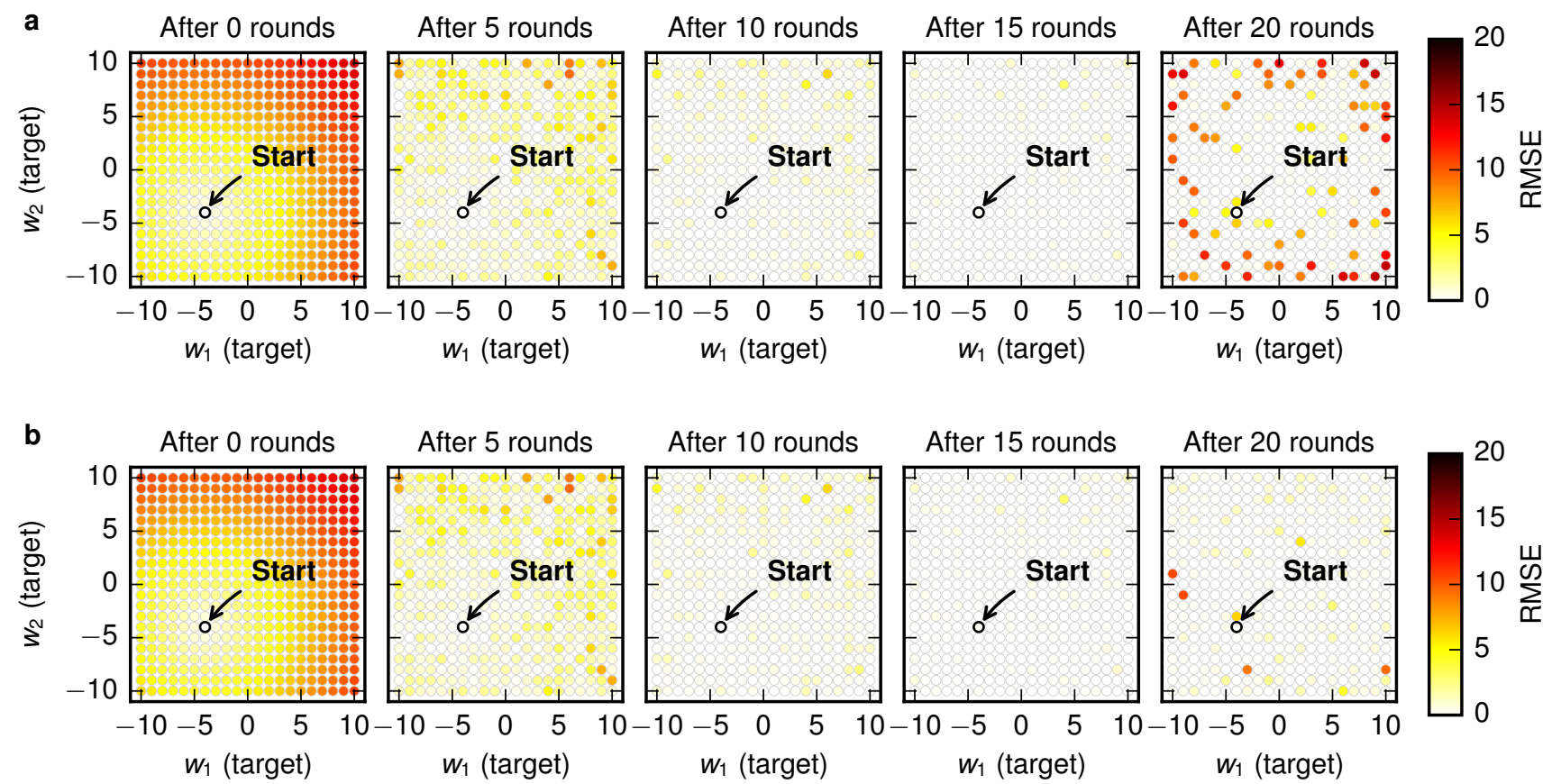

Figure S14: Scatter plots showing the learning performance over time for initial weight values $\widehat{w_{1}}=-4$ and $\widehat{w_{2}}=-4$. (a) No buffer replenishment. (b) Buffer replenished every 5 rounds.
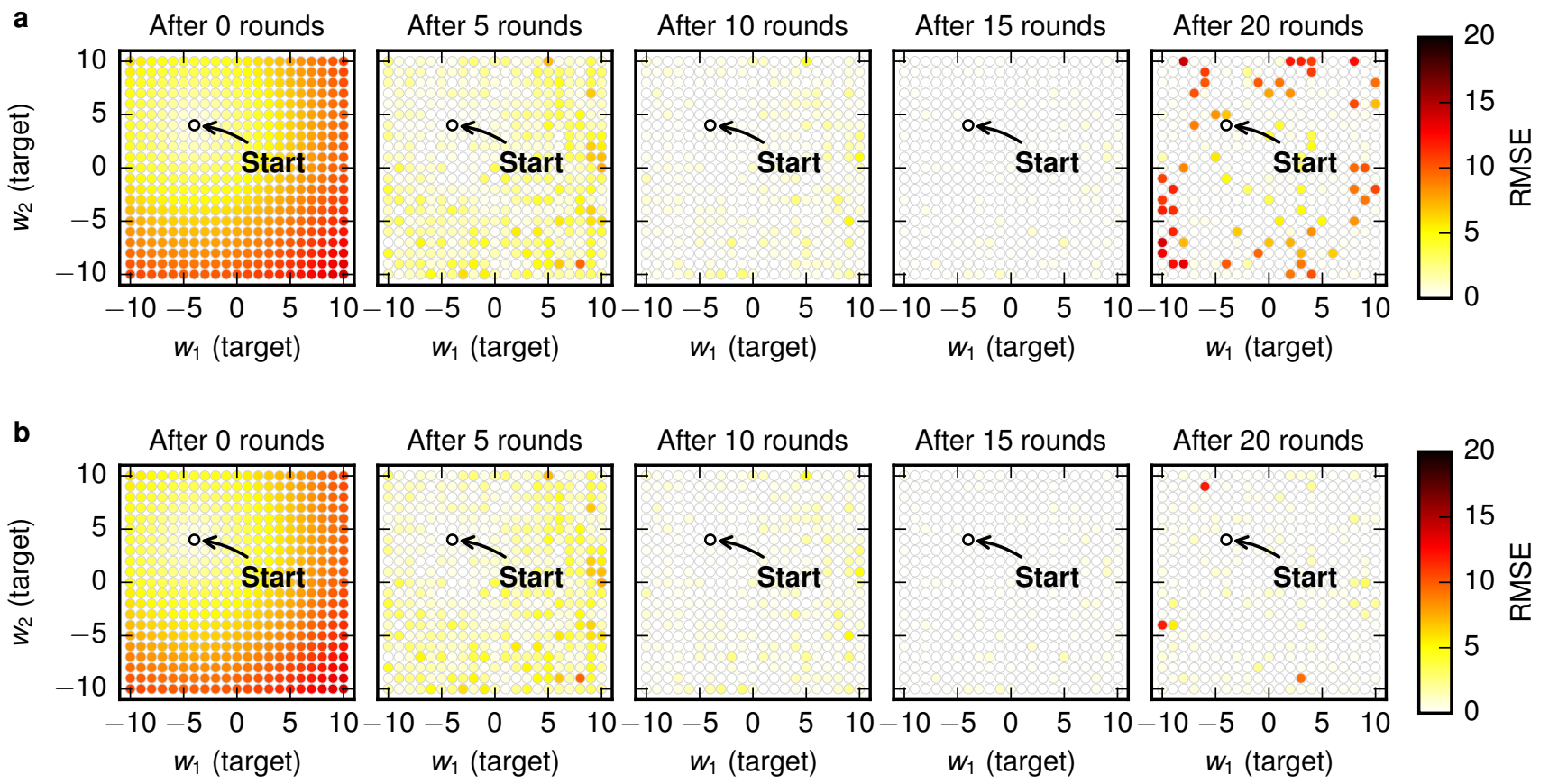

Figure S15: Scatter plots showing the learning performance over time for initial weight values $\widehat{w_{1}}=-4$ and $\widehat{w_{2}}=4$. (a) No buffer replenishment. (b) Buffer replenished every 5 rounds. 

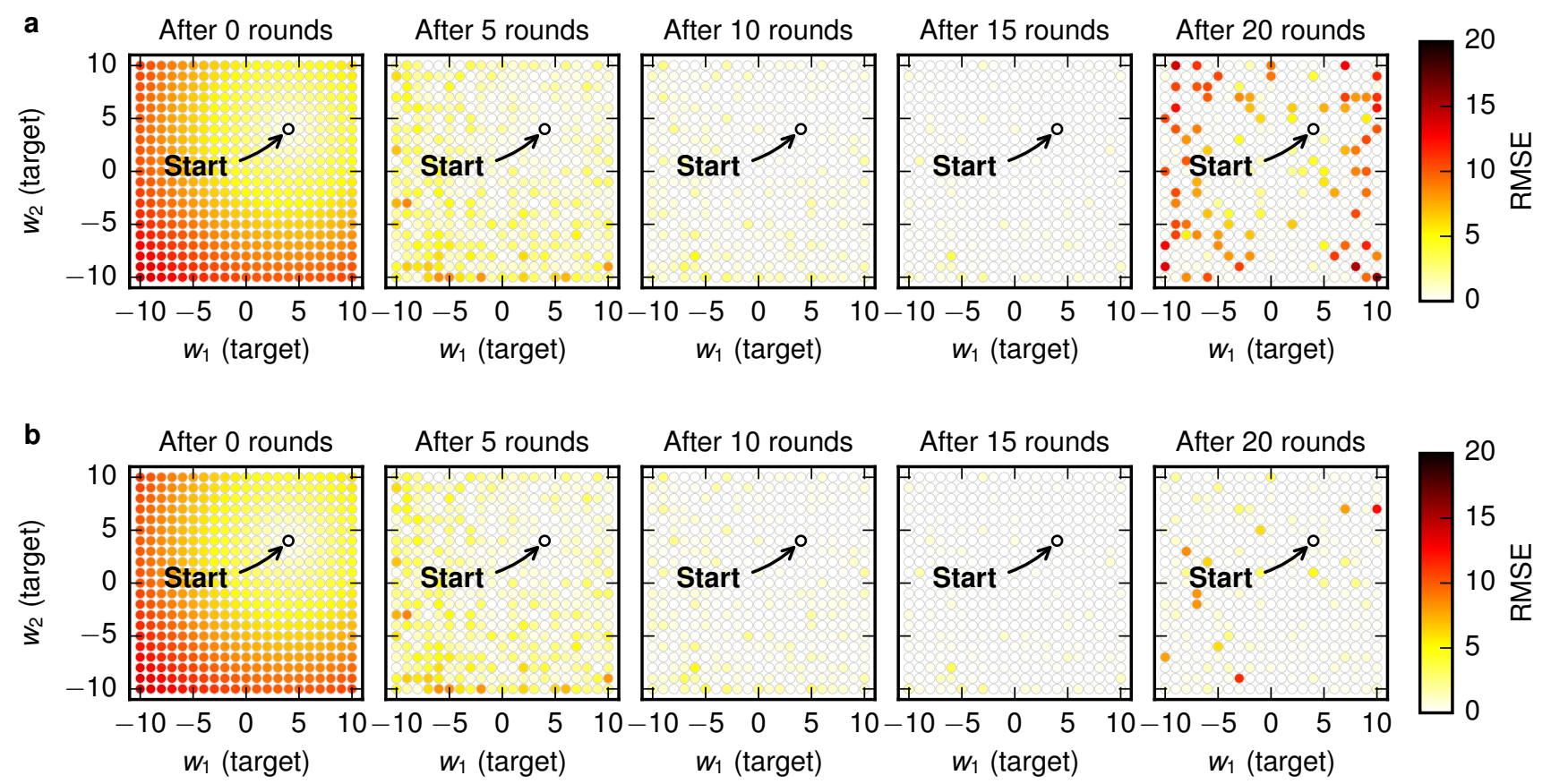

Figure S16: Scatter plots showing the learning performance over time for initial weight values $\widehat{w_{1}}=4$ and $\widehat{w_{2}}=4$. (a) No buffer replenishment. (b) Buffer replenished every 5 rounds.
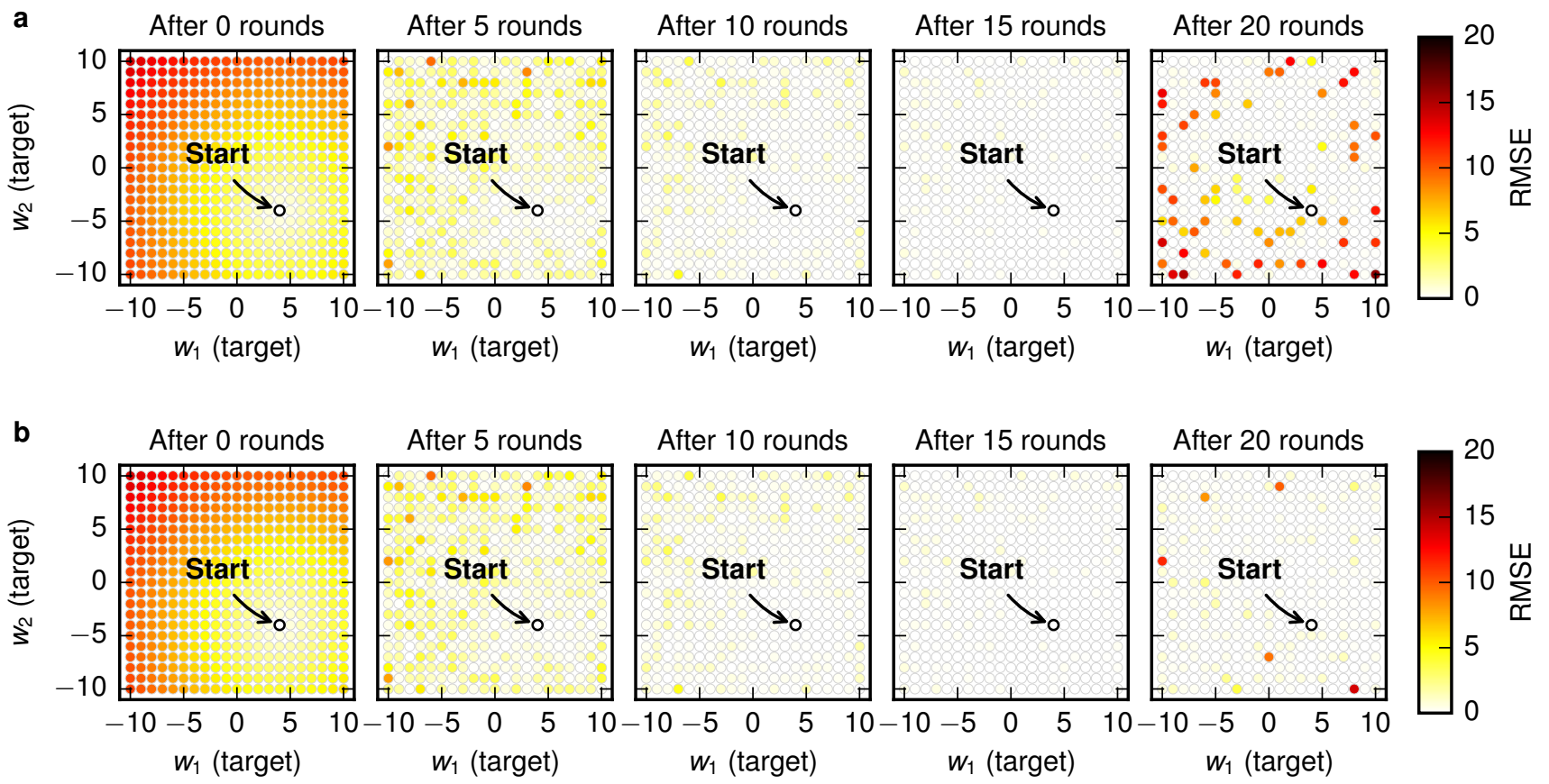

Figure S17: Scatter plots showing the learning performance over time for initial weight values $\widehat{w_{1}}=4$ and $\widehat{w_{2}}=-4$. (a) No buffer replenishment. (b) Buffer replenished every 5 rounds. 

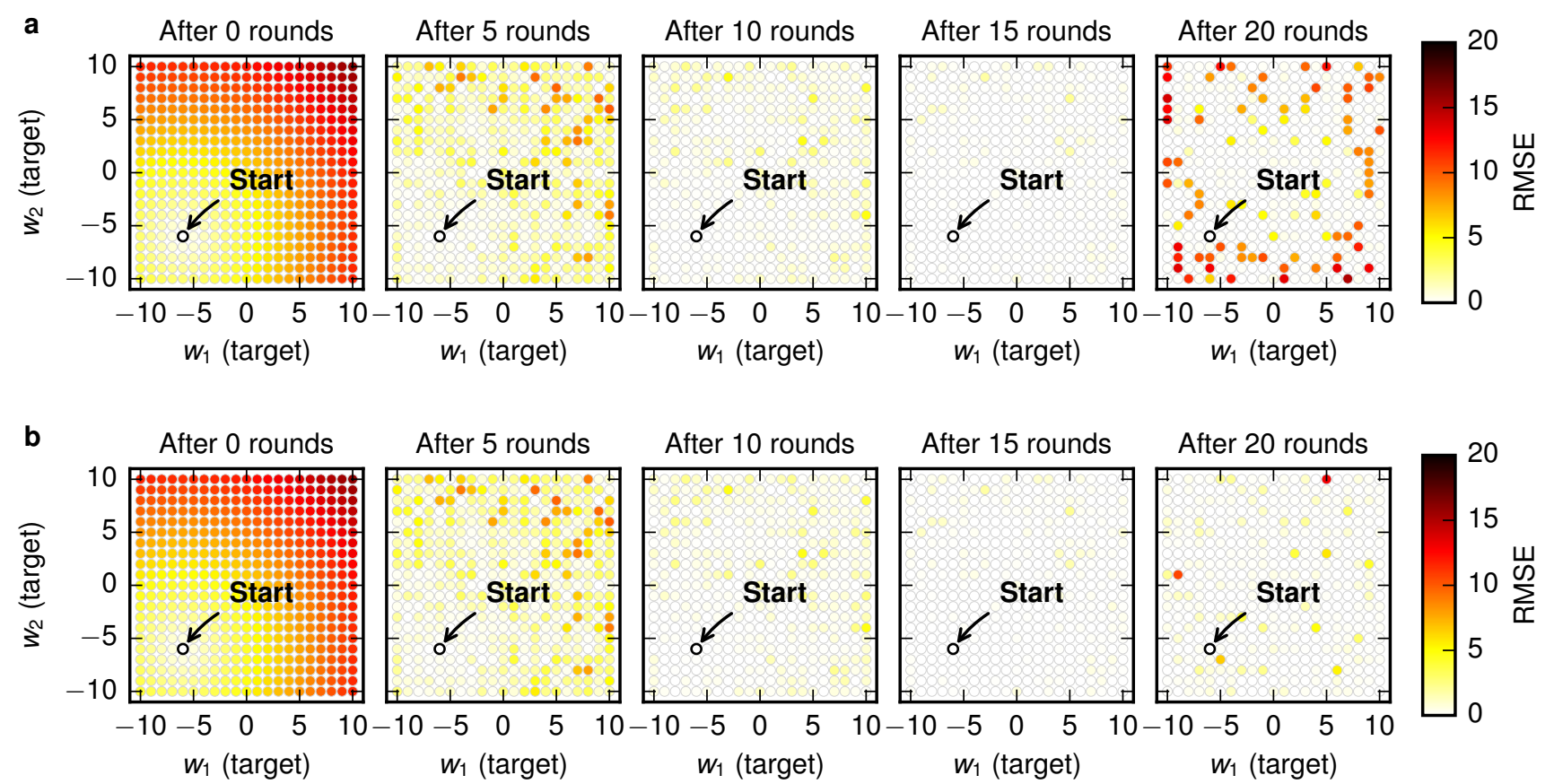

Figure S18: Scatter plots showing the learning performance over time for initial weight values $\widehat{w_{1}}=-6$ and $\widehat{w_{2}}=-6$. (a) No buffer replenishment. (b) Buffer replenished every 5 rounds.
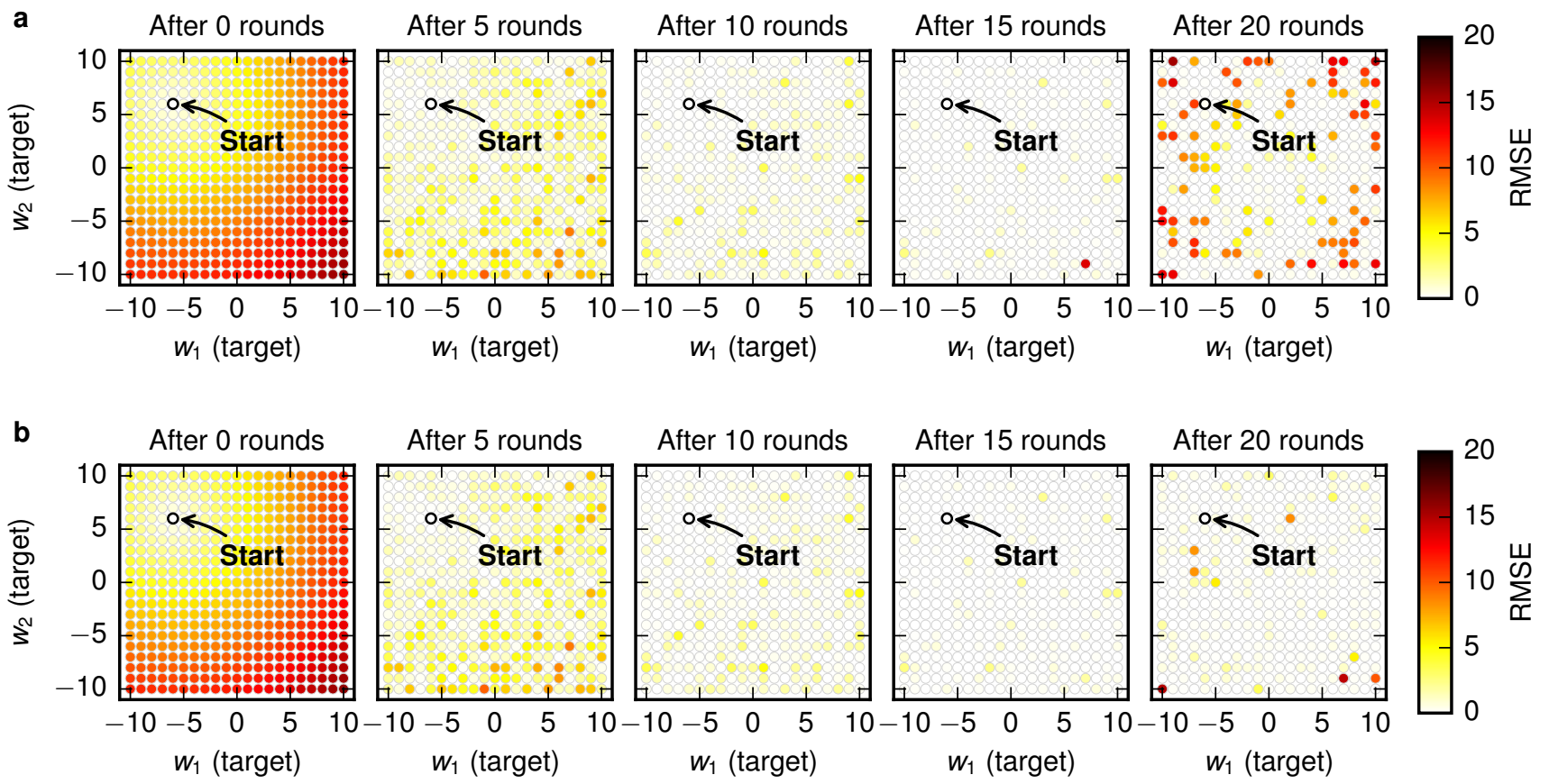

Figure S19: Scatter plots showing the learning performance over time for initial weight values $\widehat{w_{1}}=-6$ and $\widehat{w_{2}}=6$. (a) No buffer replenishment. (b) Buffer replenished every 5 rounds. 

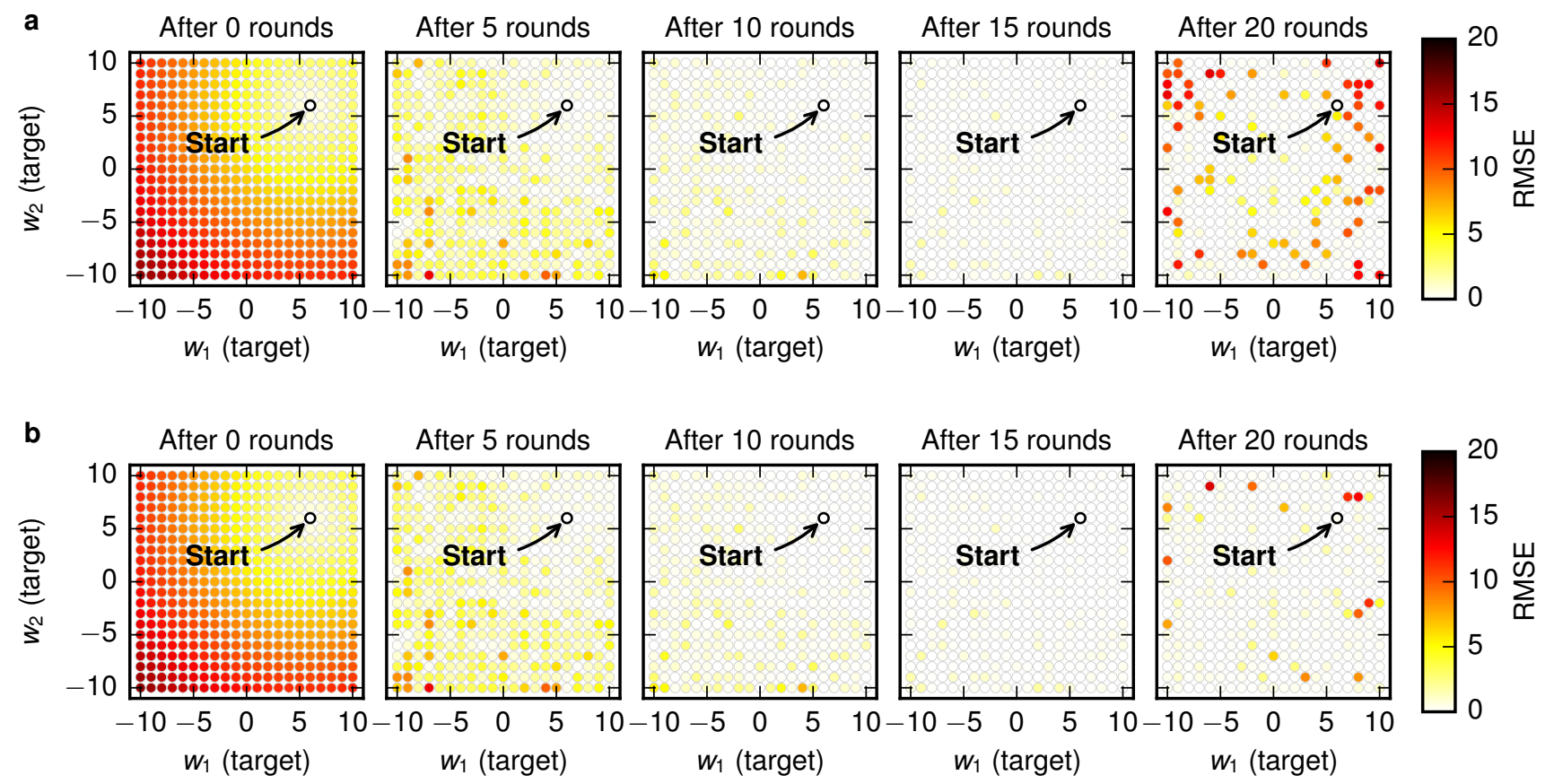

Figure S20: Scatter plots showing the learning performance over time for initial weight values $\widehat{w_{1}}=6$ and $\widehat{w_{2}}=6$. (a) No buffer replenishment. (b) Buffer replenished every 5 rounds.
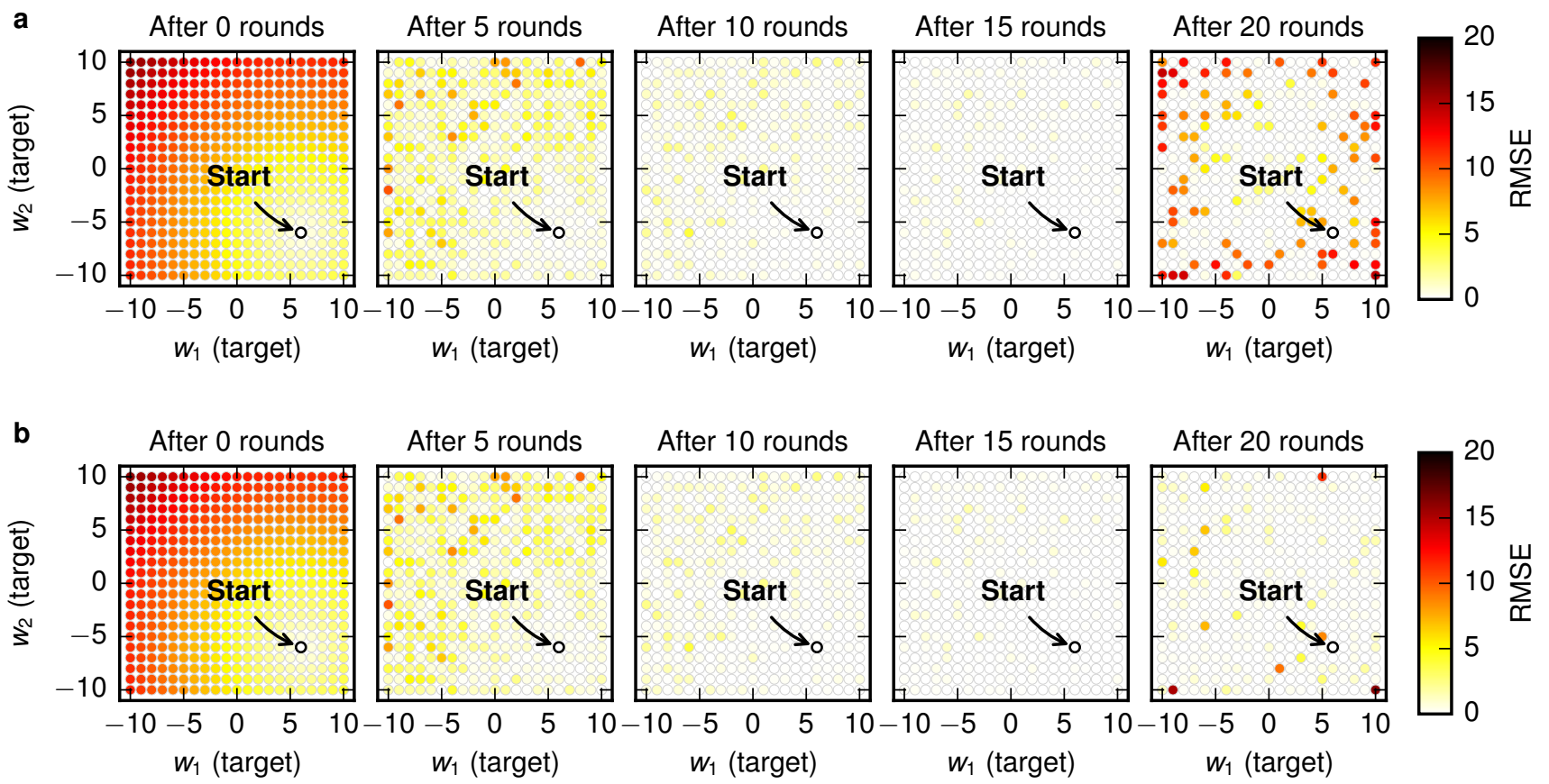

Figure S21: Scatter plots showing the learning performance over time for initial weight values $\widehat{w_{1}}=6$ and $\widehat{w_{2}}=-6$. (a) No buffer replenishment. (b) Buffer replenished every 5 rounds. 

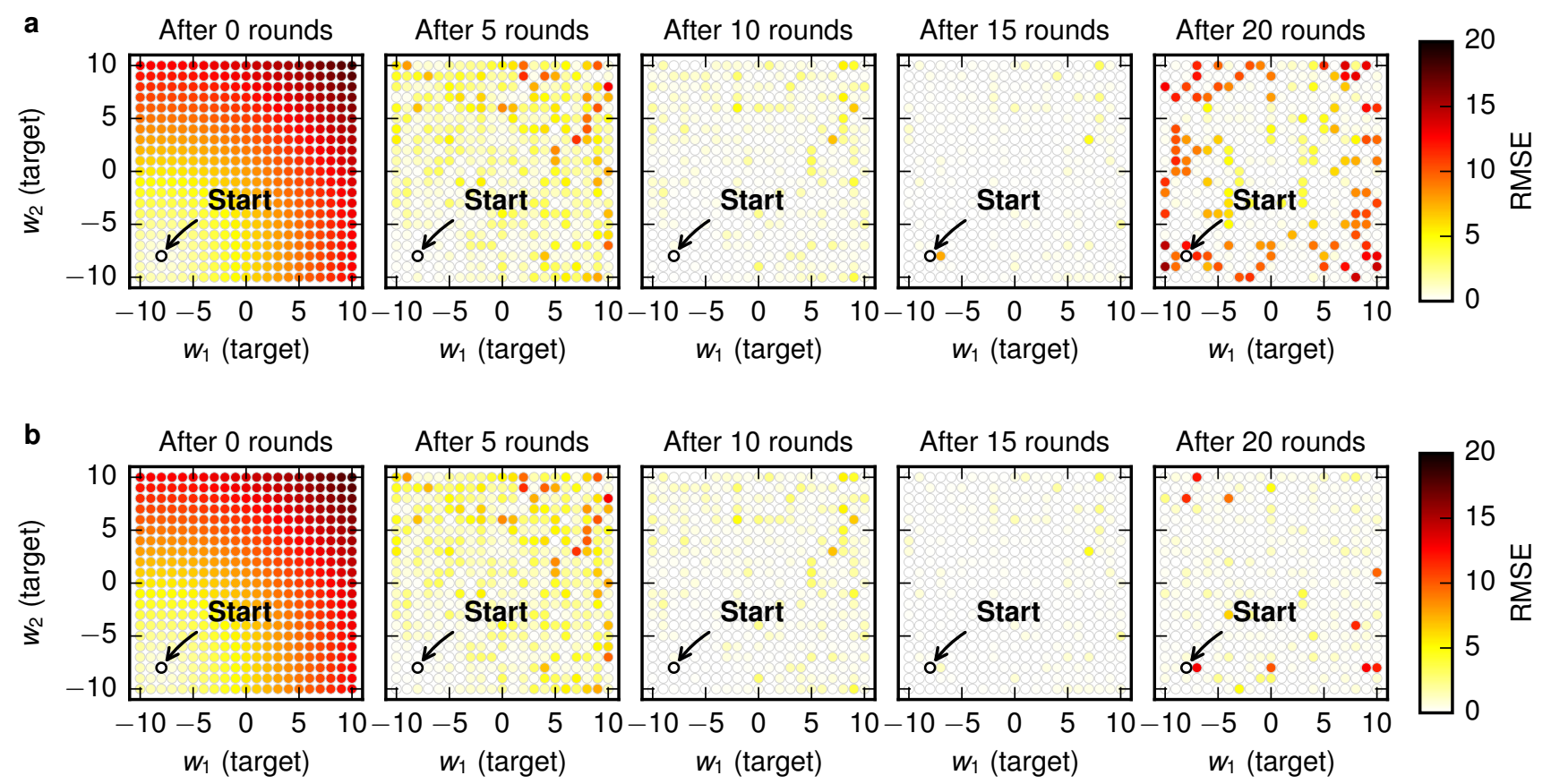

Figure S22: Scatter plots showing the learning performance over time for initial weight values $\widehat{w_{1}}=-8$ and $\widehat{w_{2}}=-8$. (a) No buffer replenishment. (b) Buffer replenished every 5 rounds.
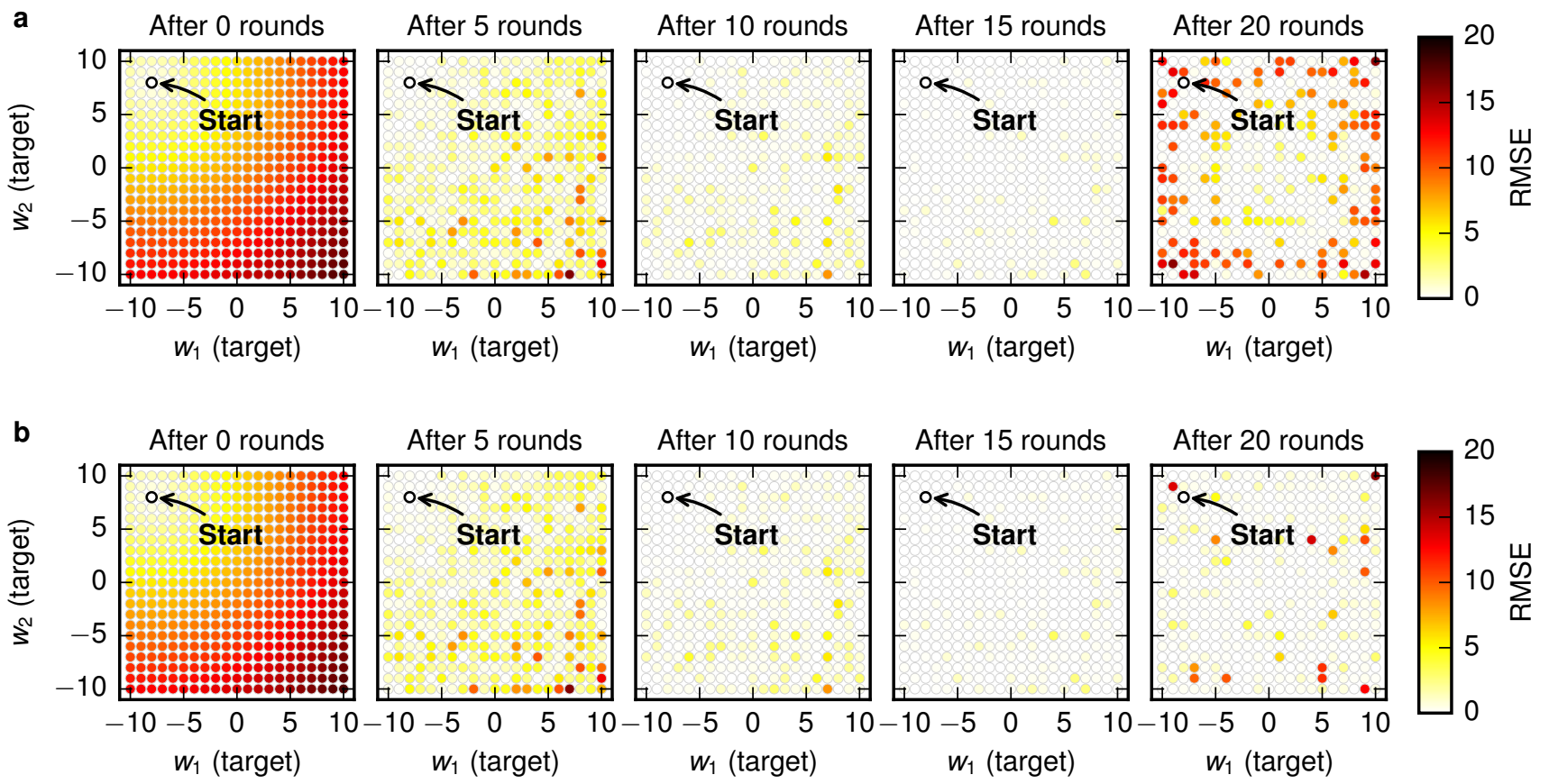

Figure S23: Scatter plots showing the learning performance over time for initial weight values $\widehat{w_{1}}=-8$ and $\widehat{w_{2}}=8$. (a) No buffer replenishment. (b) Buffer replenished every 5 rounds. 

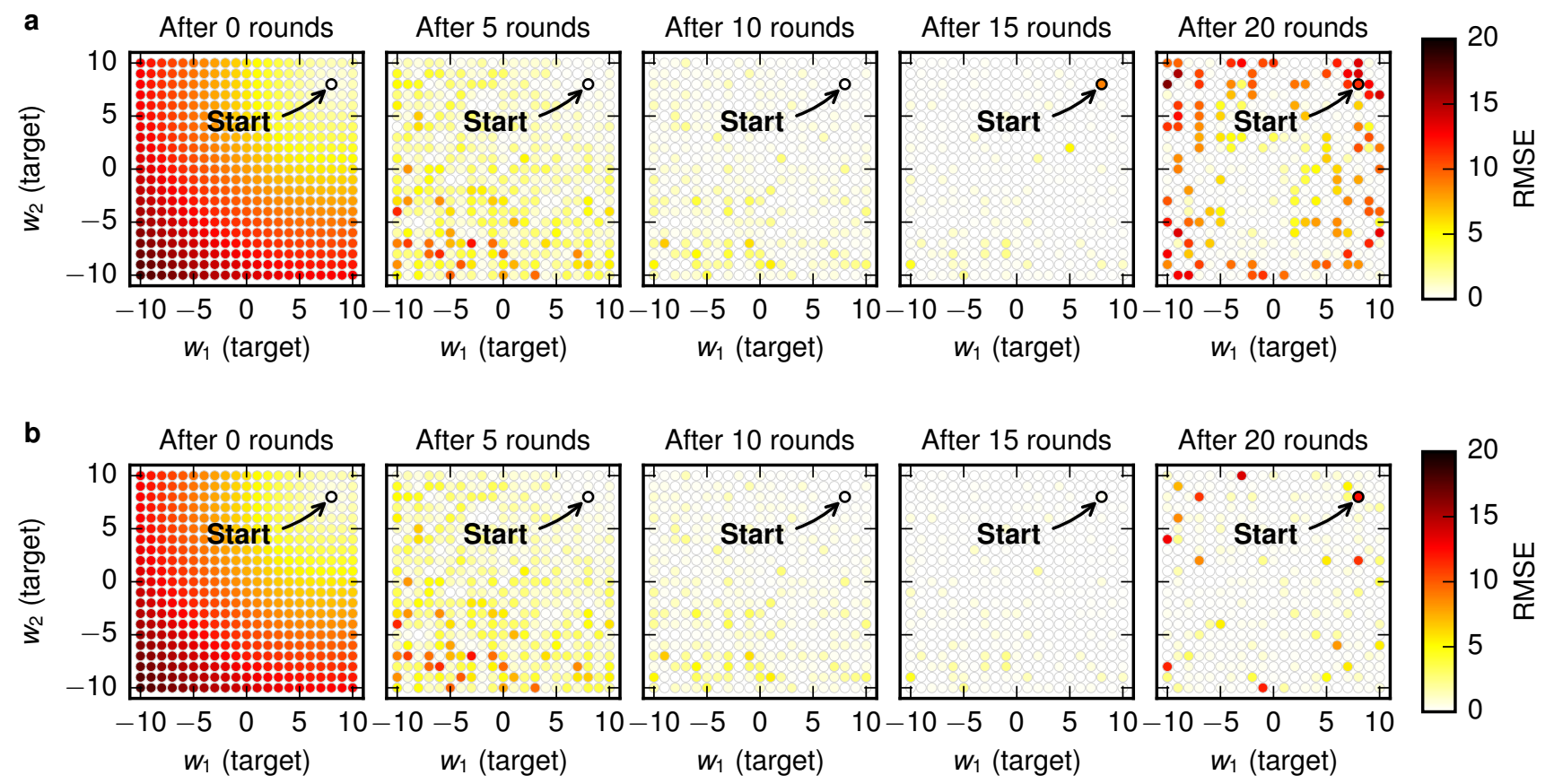

Figure S24: Scatter plots showing the learning performance over time for initial weight values $\widehat{w_{1}}=8$ and $\widehat{w_{2}}=8$. (a) No buffer replenishment. (b) Buffer replenished every 5 rounds.
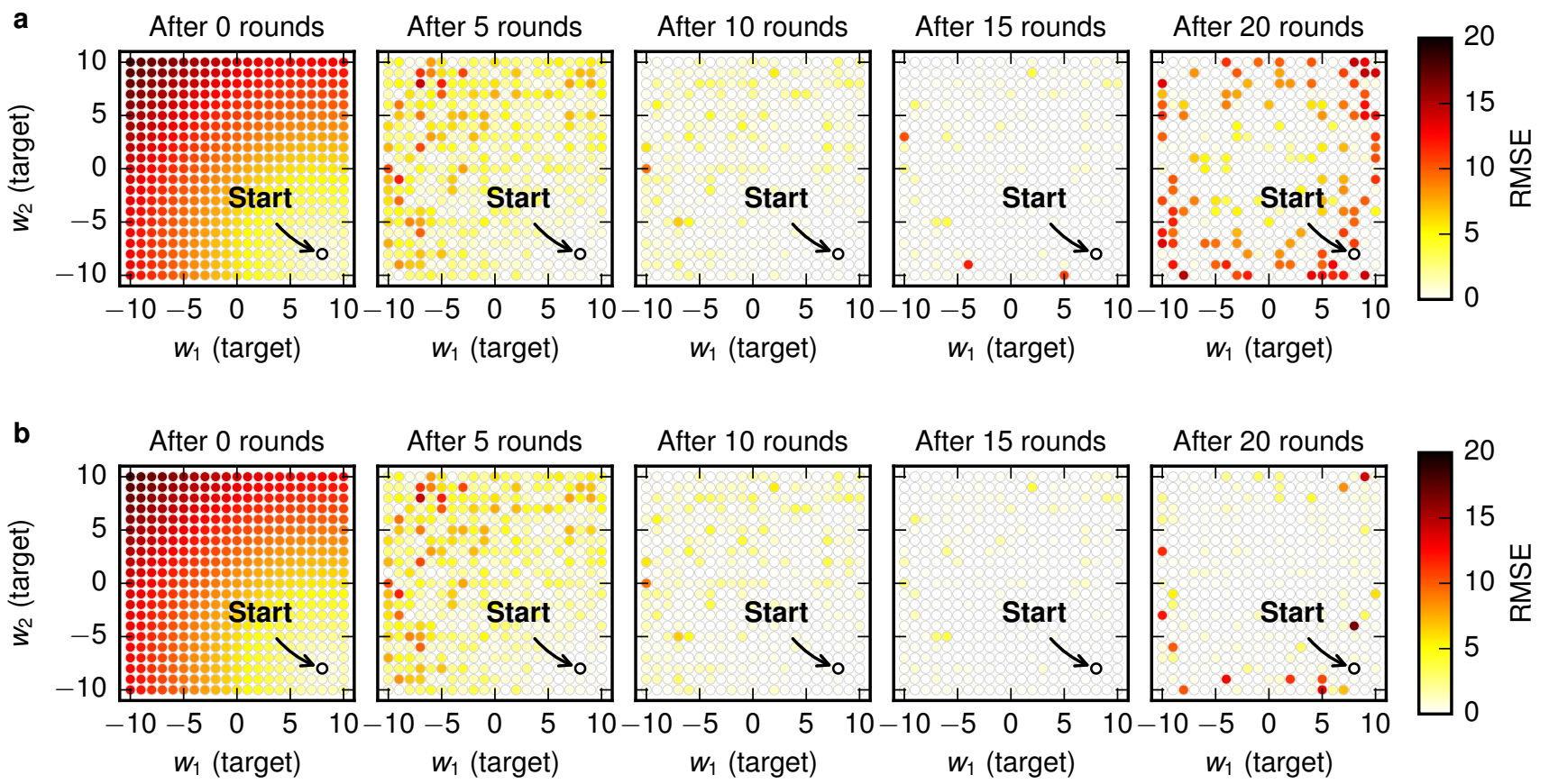

Figure S25: Scatter plots showing the learning performance over time for initial weight values $\widehat{w_{1}}=8$ and $\widehat{w_{2}}=-8$. (a) No buffer replenishment. (b) Buffer replenished every 5 rounds. 

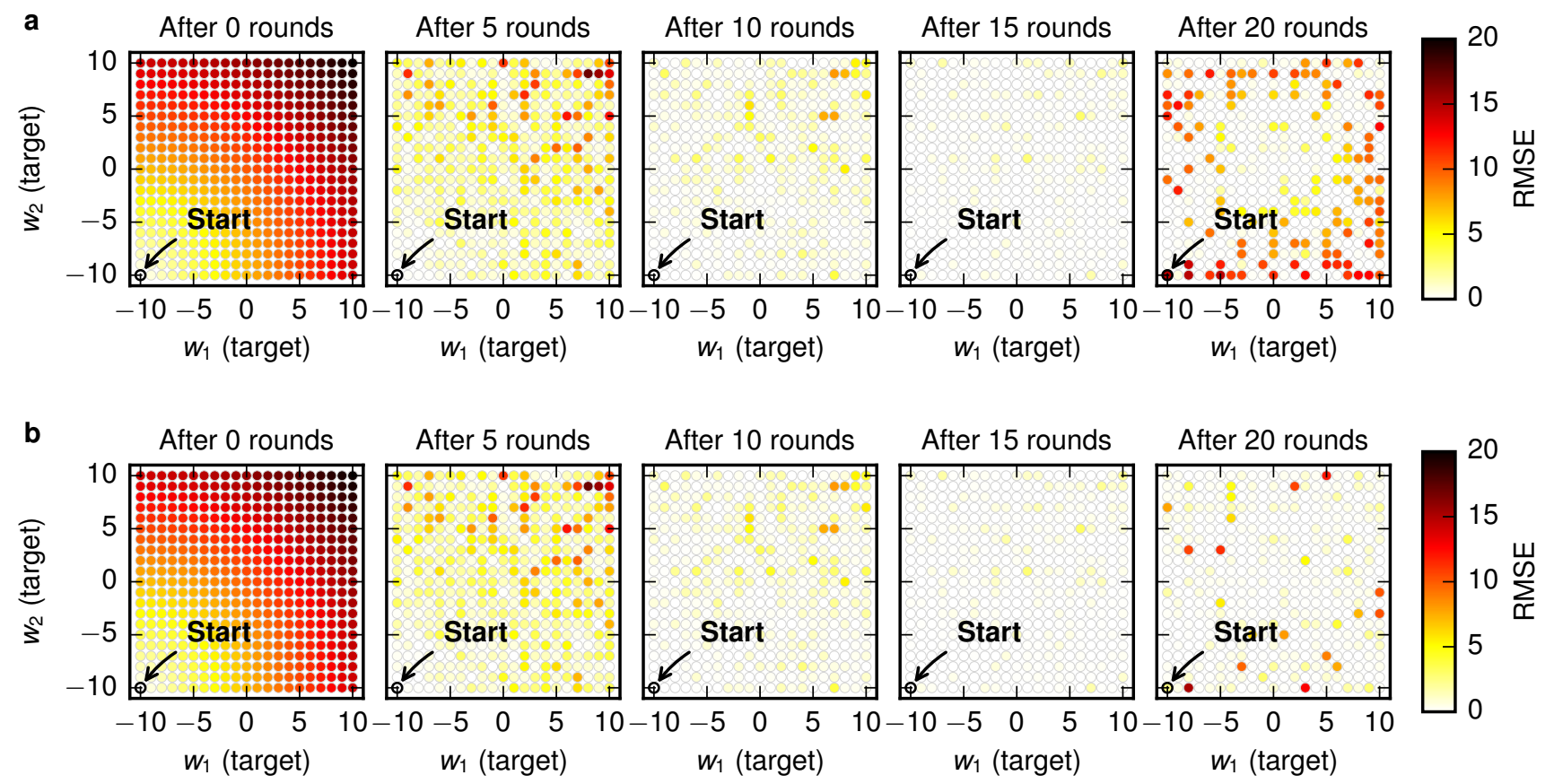

Figure S26: Scatter plots showing the learning performance over time for initial weight values $\widehat{w_{1}}=-10$ and $\widehat{w_{2}}=-10$. (a) No buffer replenishment. (b) Buffer replenished every 5 rounds.
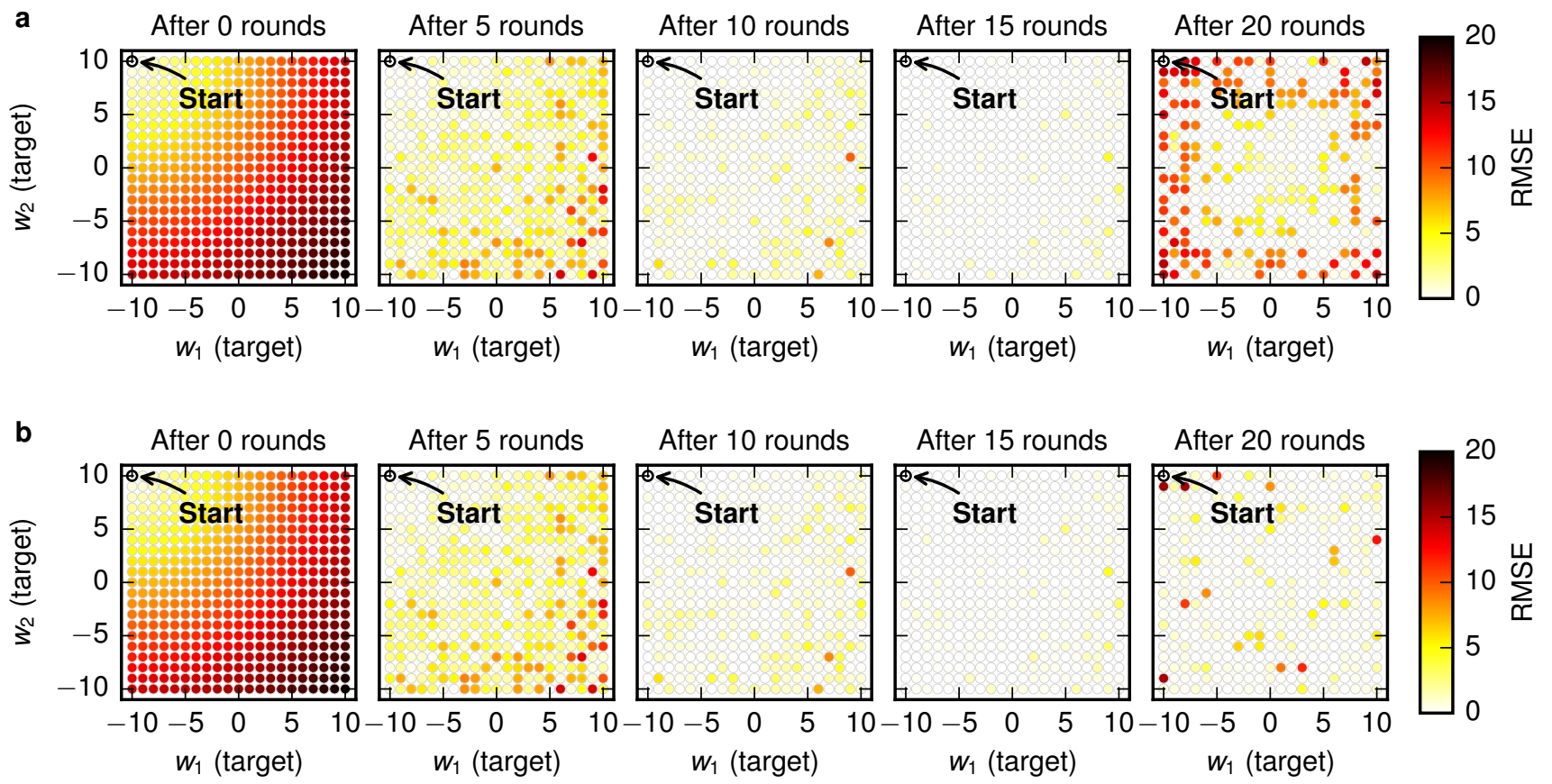

Figure S27: Scatter plots showing the learning performance over time for initial weight values $\widehat{w_{1}}=-10$ and $\widehat{w_{2}}=10$. (a) No buffer replenishment. (b) Buffer replenished every 5 rounds. 

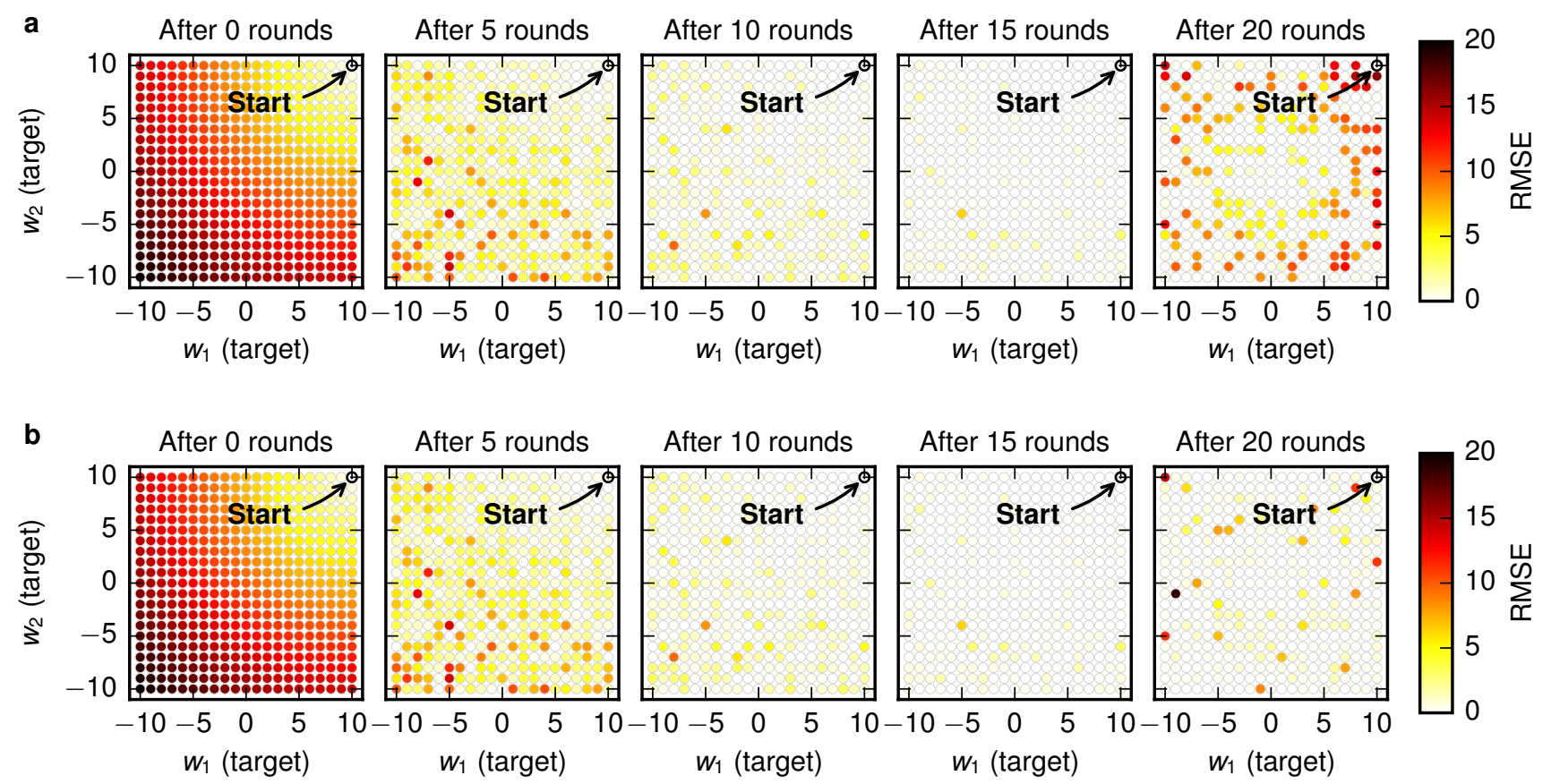

Figure S28: Scatter plots showing the learning performance over time for initial weight values $\widehat{w_{1}}=10$ and $\widehat{w_{2}}=10$. (a) No buffer replenishment. (b) Buffer replenished every 5 rounds.
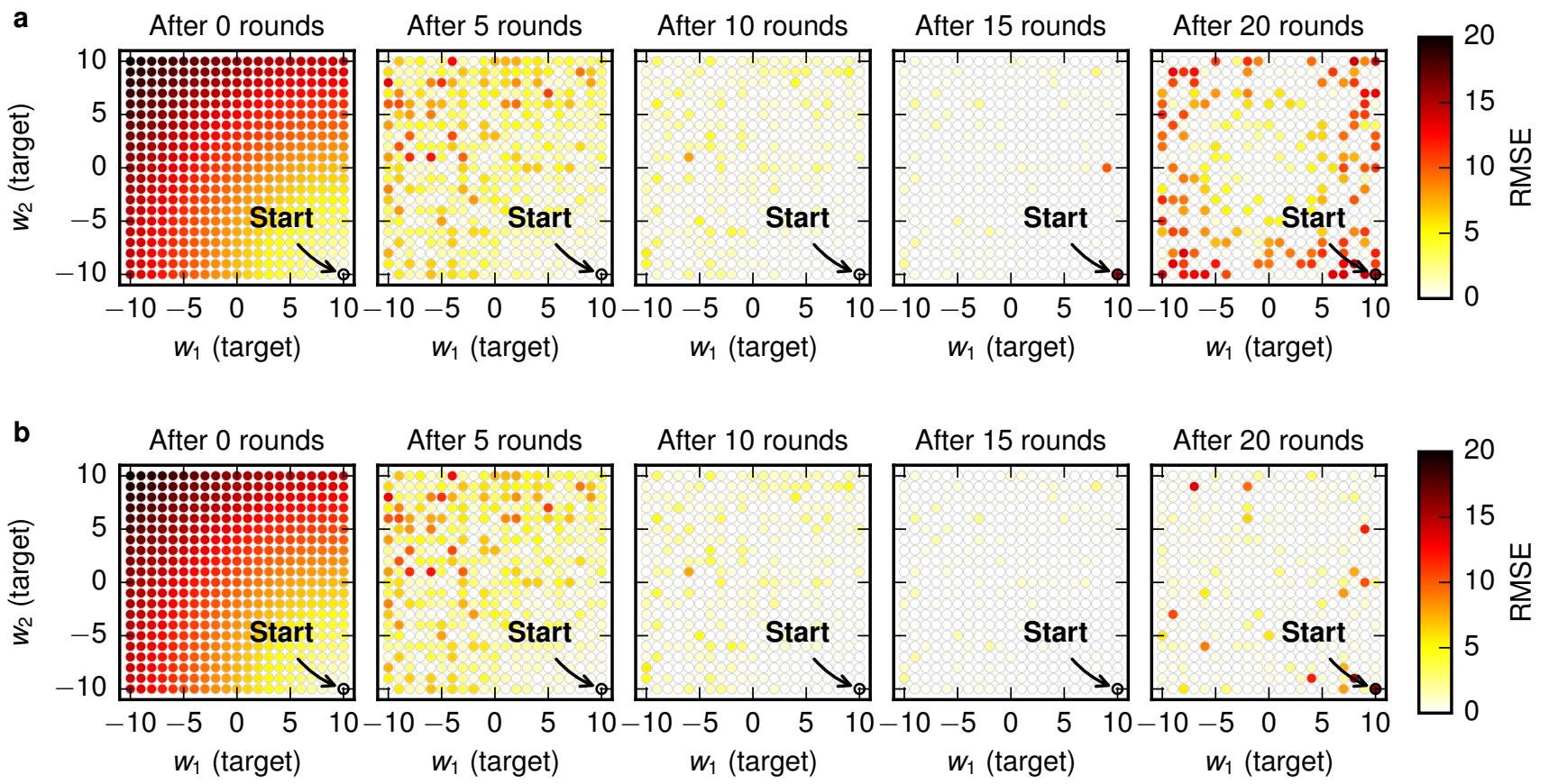

Figure S29: Scatter plots showing the learning performance over time for initial weight values $\widehat{w_{1}}=10$ and $\widehat{w_{2}}=-10$. (a) No buffer replenishment. (b) Buffer replenished every 5 rounds. 

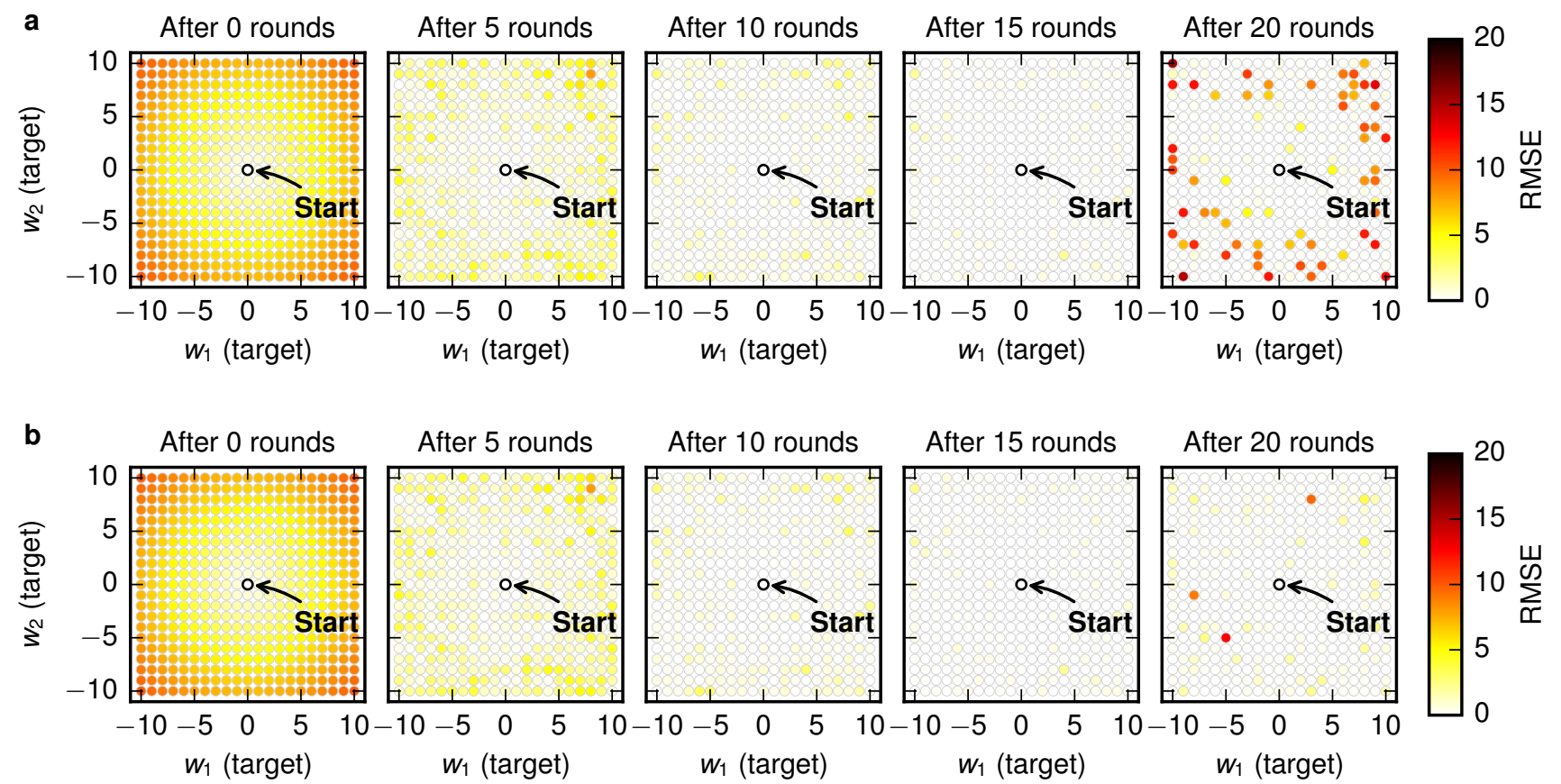

Figure S30: Scatter plots showing the learning performance over time for initial weight values $\widehat{w_{1}}=0$ and $\widehat{w_{2}}=0$. (a) No buffer replenishment. (b) Buffer replenished every 5 rounds.

\section{S4 DSD Code for Compiling the Learning Circuit}

The following code was compiled using the Visual DSD tool [1] with the "Infinite" reaction semantics selected [2].

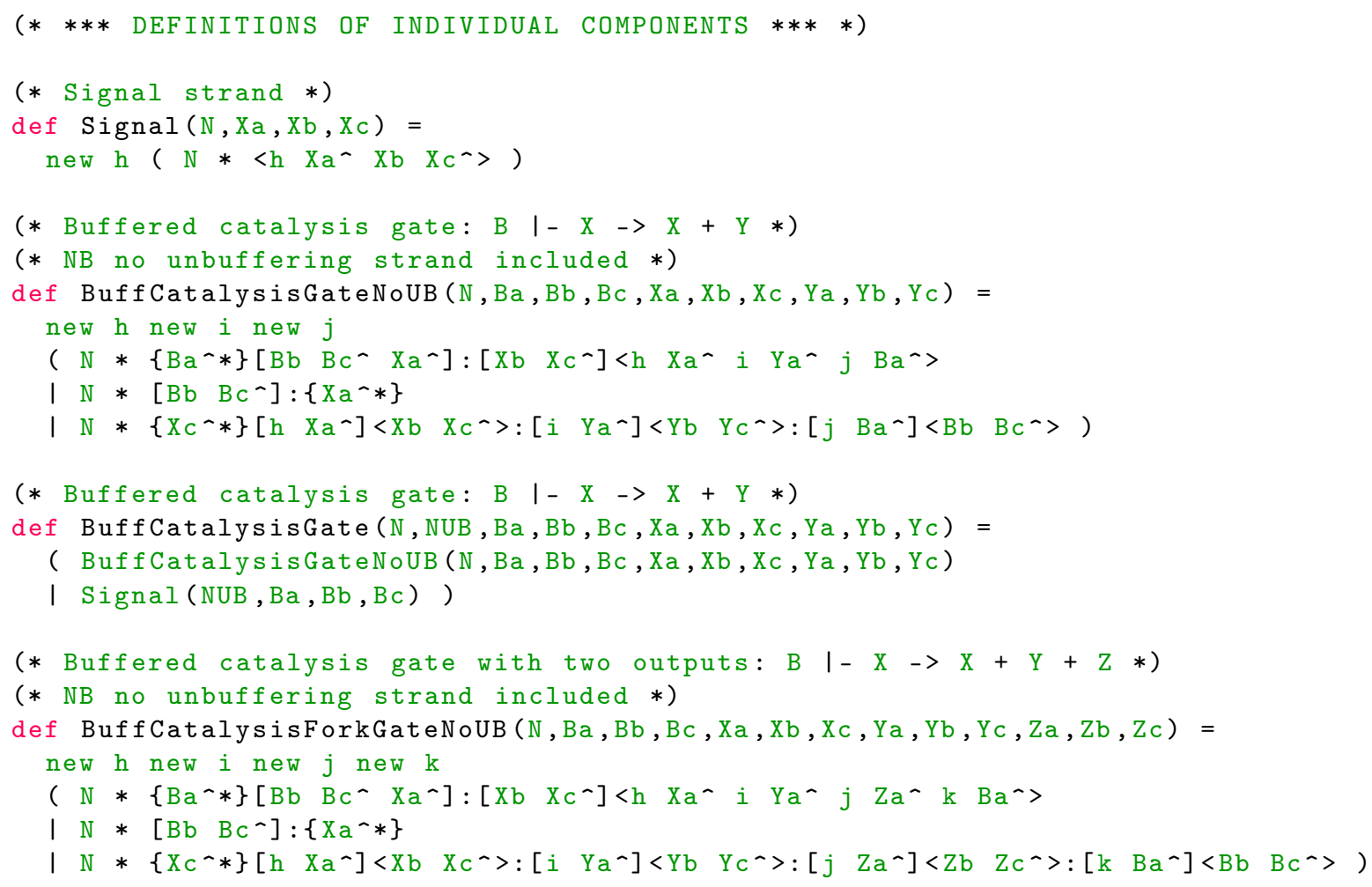




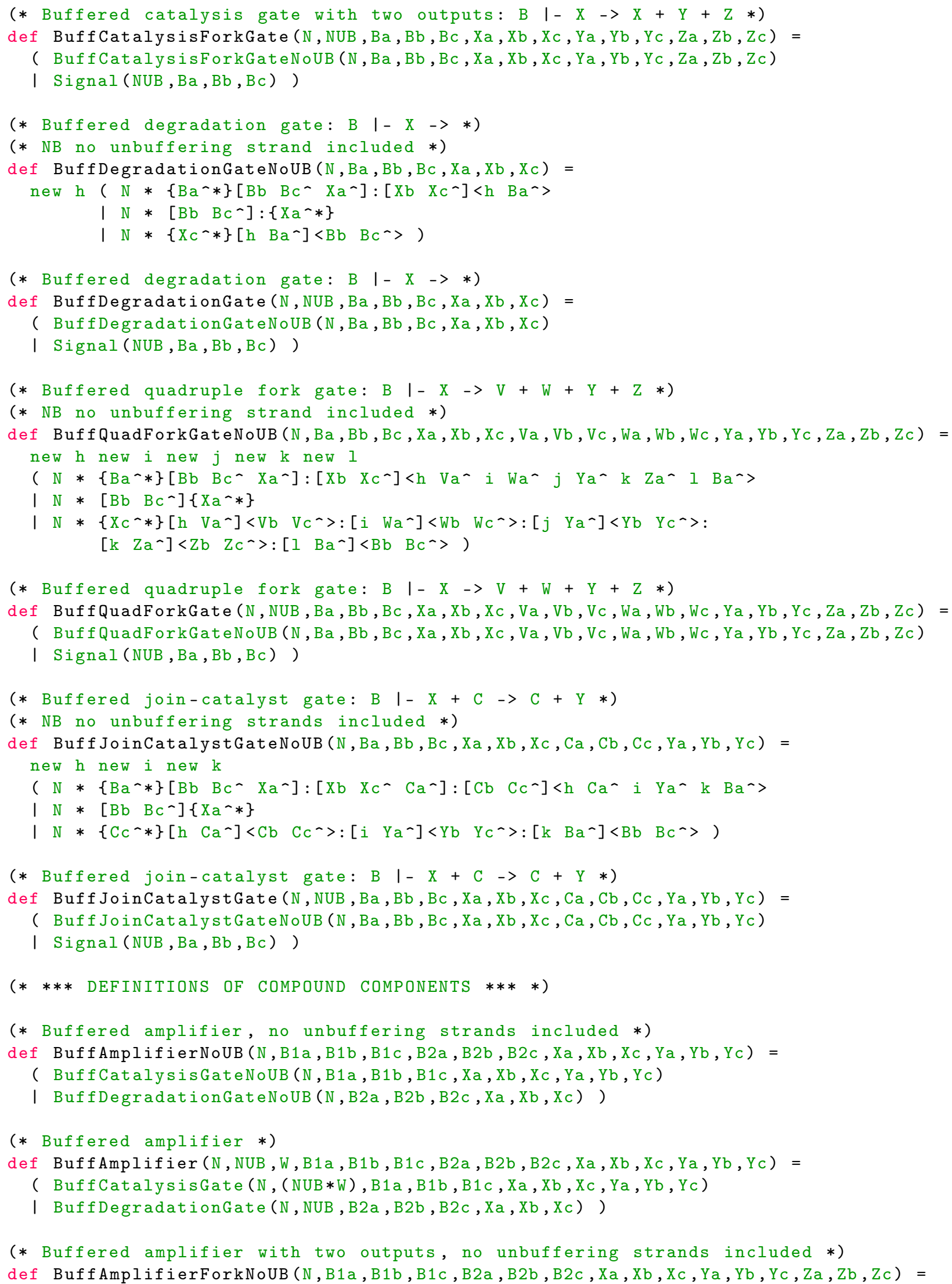


( BuffCatalysisForkGateNoUB (N, B1a, B1b, B1c, Xa, Xb, Xc, Ya, Yb, Yc, Za, Zb, Zc)

I BuffDegradationGateNoUB (N,B2a, B2b, B2c, Xa, Xb, Xc) )

(* Buffered amplifier with two outputs *)

def BuffAmplifierFork (N, NUB, W, B1a, B1b, B1c, B2a, B2b, B2c, Xa, Xb, Xc, Ya, Yb, Yc, Za, Zb, Zc) =

( BuffCatalysisForkGate (N, (NUB*W), B1a, B1b, B1c, Xa, Xb, Xc, Ya, Yb, Yc, Za, Zb, Zc)

I BuffDegradationGate (N, NUB, B2a, B2b, B2c, Xa, Xb, Xc) )

(* Buffered dual rail amplifier *)

def BuffDualRailAmplifier(N,NUB, WPlus, WMinus, XPa, XPb, XPc, XMa, XMb, XMc, $Y P a, Y P b, Y P c, Y M a, Y M b, Y M c, K P 1 a, K P 1 b, K P 1 c, K P 2 a, K P 2 b, K P 2 c$, $\mathrm{KM} 1 \mathrm{a}, \mathrm{KM} 1 \mathrm{~b}, \mathrm{KM} 1 \mathrm{c}, \mathrm{KM} 2 \mathrm{a}, \mathrm{KM} 2 \mathrm{~b}, \mathrm{KM} 2 \mathrm{c}, \mathrm{B} 1 \mathrm{a}, \mathrm{B} 1 \mathrm{~b}, \mathrm{~B} 1 \mathrm{c}, \mathrm{B} 2 \mathrm{a}, \mathrm{B} 2 \mathrm{~b}, \mathrm{~B} 2 \mathrm{c}, \mathrm{B} 3 \mathrm{a}, \mathrm{B} 3 \mathrm{~b}, \mathrm{~B} 3 \mathrm{c}$,

$\mathrm{B} 4 \mathrm{a}, \mathrm{B} 4 \mathrm{~b}, \mathrm{~B} 4 \mathrm{c}, \mathrm{B} 5 \mathrm{a}, \mathrm{B} 5 \mathrm{~b}, \mathrm{~B} 5 \mathrm{c}, \mathrm{B} 6 \mathrm{a}, \mathrm{B} 6 \mathrm{~b}, \mathrm{~B} 6 \mathrm{c}, \mathrm{B} 7 \mathrm{a}, \mathrm{B} 7 \mathrm{~b}, \mathrm{~B} 7 \mathrm{c}, \mathrm{B} 8 \mathrm{a}, \mathrm{B} 8 \mathrm{~b}, \mathrm{~B} 8 \mathrm{c})=$

new XP1a new XP1b new XP1c new XP2a new XP2b new XP2c

new XM1a new XM1b new XM1c new XM2a new XM2b new XM2c

new B9a new B9b new B9c new B10a new B10b new B10c

( BuffQuadForkGate (N, NUB, B9a, B9b, B9c, XPa, XPb, XPc, XP1a, XP1b, XP1c, $X P 2 a, X P 2 b, X P 2 c, K P 1 a, K P 1 b, K P 1 c, K P 2 a, K P 2 b, K P 2 c$ )

I BuffAmplifier(N, NUB, WPIus, B1a,B1b,B1c, B2a, B2b, B2c, XP1a, XP1b, XP1c, YPa, YPb, YPc)

I BuffAmplifier (N, NUB, WMinus, B3a, B3b, B3c, B4a, B4b, B4c, XP2a, XP2b, XP2c, YMa, YMb, YMc)

I BuffQuadForkGate (N, NUB, B10a, B10b, B10c, XMa, XMb , XMc, XM1a, XM1b , XM1c, $\mathrm{XM} 2 \mathrm{a}, \mathrm{XM} 2 \mathrm{~b}, \mathrm{XM} 2 \mathrm{c}, \mathrm{KM} 1 \mathrm{a}, \mathrm{KM} 1 \mathrm{~b}, \mathrm{KM} 1 \mathrm{c}, \mathrm{KM} 2 \mathrm{a}, \mathrm{KM} 2 \mathrm{~b}, \mathrm{KM} 2 \mathrm{c}$ )

I BuffAmplifier(N, NUB, WPlus, B5a, B5b, B5c, B6a, B6b, B6c, XM1a, XM1b, XM1c, YMa, YMb, YMc)

I BuffAmplifier(N, NUB, WMinus, B7a, B7b,B7c, B8a, B8b, B8c, XM2a, XM2b, XM2c, YPa, YPb, YPc) )

(* Buffered dual rail subtractor *)

def BuffDualRailSubtractor ( $N$, NUB, DPa, DPb , DPc, DMa, DMb, DMc, YPa, YPb, YPc, YMa, YMb, YMc) = new B1a new B1b new B1c new B2a new B2b new B2c new B3a new B3b new B3c new B4a new B4b new B4C

( BuffJoinCatalystGate ( N, NUB, B1a, B1b, B1c, YPa, YPb, YPc, DPa, DPb, DPc, DMa, DMb, DMc)

I BuffJoinCatalystGate (N, NUB, B2a, B2b, B2c, YMa, YMb, YMc, DPa, DPb, DPc, DPa, DPb, DPc)

I BuffJoinCatalystGate (N, NUB, B3a, B3b, B3c, YMa, YMb, YMc, DMa, DMb, DMc, DPa, DPb, DPc)

I BuffJoinCatalystGate (N, NUB, B4a, B4b, B4c, YPa, YPb, YPc, DMa, DMb, DMc, DMa, DMb, DMc) )

(* Double multiplier with two outputs *)

(* Computes $\mathrm{W}=\mathrm{Z}=$ alpha $*[\mathrm{X}] *[\mathrm{Y}]$, with constant input $\operatorname{signal} \mathrm{C}=1 *$ )

(* Learning rate, alpha = AlphaM *)

def BuffDoubleMultiplierFork (N, NUB, M, AlphaM, B1a, B1b, B1c, B2a, B2b, B2c, $\mathrm{Ca}, \mathrm{Cb}, \mathrm{Cc}, \mathrm{Xa}, \mathrm{Xb}, \mathrm{Xc}, \mathrm{Ya}, \mathrm{Yb}, \mathrm{Yc}, \mathrm{Za}, \mathrm{Zb}, \mathrm{Zc}, \mathrm{Wa}, \mathrm{Wb}, \mathrm{Wc})=$ new B3a new B3b new B3c new B4a new B4b new B4c new $B 5 a$ new $B 5 b$ new $B 5 c$ new $B 6 a$ new $B 6 b$ new $B 6 c$ ( BuffAmplifierForkNoUB (N,B1a,B1b,B1c,B2a, B2b, B2c, Xa, Xb, Xc, Za, Zb, Zc, Wa, Wb, Wc)

I BuffAmplifier (N, NUB, AlphaM, B3a, B3b, B3c,B4a, B4b, B4c, Ya, Yb, Yc, B1a, B1b, B1c)

I BuffAmplifier(N, NUB, M, B5a, B5b, B5c, B6a, B6b, B6c, Ca, Cb, Cc, B2a, B2b, B2c) )

(* Two-input predictor subcircuit *)

def TwoInputPredictorSubcircuit (N, NUB, W1Plus, W1Minus, W2Plus, W2Minus, $\mathrm{X} 1 \mathrm{~Pa}, \mathrm{X} 1 \mathrm{~Pb}, \mathrm{X} 1 \mathrm{Pc}, \mathrm{X} 1 \mathrm{Ma}, \mathrm{X} 1 \mathrm{Mb}, \mathrm{X} 1 \mathrm{Mc}, \mathrm{X} 2 \mathrm{~Pa}, \mathrm{X} 2 \mathrm{~Pb}, \mathrm{X} 2 \mathrm{Pc}, \mathrm{X} 2 \mathrm{Ma}, \mathrm{X} 2 \mathrm{Mb}, \mathrm{X} 2 \mathrm{Mc}$, $\mathrm{YPa}, \mathrm{YPb}, \mathrm{YPc}, \mathrm{YMa}, \mathrm{YMb}, \mathrm{YMc}, \mathrm{DPa}, \mathrm{DPb}, \mathrm{DPc}, \mathrm{DMa}, \mathrm{DMb}, \mathrm{DMc}$, K1P1a, K1P1b, K1P1c,K1P2a, K1P2b, K1P2c,K1M1a, K1M1b, K1M1c, K1M2a, K1M2b, K1M2c, $\mathrm{K} 2 \mathrm{P} 1 \mathrm{a}, \mathrm{K} 2 \mathrm{P} 1 \mathrm{~b}, \mathrm{~K} 2 \mathrm{P} 1 \mathrm{c}, \mathrm{K} 2 \mathrm{P} 2 \mathrm{a}, \mathrm{K} 2 \mathrm{P} 2 \mathrm{~b}, \mathrm{~K} 2 \mathrm{P} 2 \mathrm{c}, \mathrm{K} 2 \mathrm{M} 1 \mathrm{a}, \mathrm{K} 2 \mathrm{M} 1 \mathrm{~b}, \mathrm{~K} 2 \mathrm{M} 1 \mathrm{c}, \mathrm{K} 2 \mathrm{M} 2 \mathrm{a}, \mathrm{K} 2 \mathrm{M} 2 \mathrm{~b}, \mathrm{~K} 2 \mathrm{M} 2 \mathrm{c}$, B11a, B11b, B11c, B12a, B12b, B12c, B13a, B13b, B13c, B14a, B14b, B14c, $\mathrm{B} 15 \mathrm{a}, \mathrm{B} 15 \mathrm{~b}, \mathrm{~B} 15 \mathrm{c}, \mathrm{B} 16 \mathrm{a}, \mathrm{B} 16 \mathrm{~b}, \mathrm{~B} 16 \mathrm{c}, \mathrm{B} 17 \mathrm{a}, \mathrm{B} 17 \mathrm{~b}, \mathrm{~B} 17 \mathrm{c}, \mathrm{B} 18 \mathrm{a}, \mathrm{B} 18 \mathrm{~b}, \mathrm{~B} 18 \mathrm{c}$, B21a, B21b, B21c, B22a, B22b, B22c, B23a, B23b, B23c, B24a, B24b, B24c, $\mathrm{B} 25 \mathrm{a}, \mathrm{B} 25 \mathrm{~b}, \mathrm{~B} 25 \mathrm{c}, \mathrm{B} 26 \mathrm{a}, \mathrm{B} 26 \mathrm{~b}, \mathrm{~B} 26 \mathrm{c}, \mathrm{B} 27 \mathrm{a}, \mathrm{B} 27 \mathrm{~b}, \mathrm{~B} 27 \mathrm{c}, \mathrm{B} 28 \mathrm{a}, \mathrm{B} 28 \mathrm{~b}, \mathrm{~B} 28 \mathrm{c})=$

( BuffDualRailAmplifier (N, NUB, W1Plus, W1Minus, $\mathrm{X} 1 \mathrm{~Pa}, \mathrm{X} 1 \mathrm{~Pb}, \mathrm{X} 1 \mathrm{Pc}, \mathrm{X} 1 \mathrm{Ma}, \mathrm{X} 1 \mathrm{Mb}, \mathrm{X} 1 \mathrm{Mc}, \mathrm{YPa}, \mathrm{YPb}, \mathrm{YPc}, \mathrm{YMa}, \mathrm{YMb}, \mathrm{YMc}$, K1P1a, K1P1b, K1P1c,K1P2a, K1P2b, K1P2c,K1M1a, K1M1b, K1M1c, K1M2a, K1M2b, K1M2c, B11a, B11b, B11c,B12a, B12b, B12c,B13a, B13b, B13c,B14a, B14b, B14c, 
B15a, B15b, B15c,B16a, B16b, B16c, B17a, B17b, B17c, B18a, B18b, B18c)

I BuffDualRailAmplifier(N, NUB, W2Plus, W2Minus,

$\mathrm{X} 2 \mathrm{~Pa}, \mathrm{X} 2 \mathrm{~Pb}, \mathrm{X} 2 \mathrm{Pc}, \mathrm{X} 2 \mathrm{Ma}, \mathrm{X} 2 \mathrm{Mb}, \mathrm{X} 2 \mathrm{Mc}, \mathrm{YPa}, \mathrm{YPb}, \mathrm{YPc}, \mathrm{YMa}, \mathrm{YMb}, \mathrm{YMc}$,

$\mathrm{K} 2 \mathrm{P} 1 \mathrm{a}, \mathrm{K} 2 \mathrm{P} 1 \mathrm{~b}, \mathrm{~K} 2 \mathrm{P} 1 \mathrm{c}, \mathrm{K} 2 \mathrm{P} 2 \mathrm{a}, \mathrm{K} 2 \mathrm{P} 2 \mathrm{~b}, \mathrm{~K} 2 \mathrm{P} 2 \mathrm{c}, \mathrm{K} 2 \mathrm{M} 1 \mathrm{a}, \mathrm{K} 2 \mathrm{M} 1 \mathrm{~b}, \mathrm{~K} 2 \mathrm{M} 1 \mathrm{c}, \mathrm{K} 2 \mathrm{M} 2 \mathrm{a}, \mathrm{K} 2 \mathrm{M} 2 \mathrm{~b}, \mathrm{~K} 2 \mathrm{M} 2 \mathrm{c}$,

B21a, B21b, B21c, B22a, B22b, B22c, B23a, B23b, B23c, B24a, B24b, B24c,

B25a, B25b, B25c, B26a, B26b, B26c, B27a, B27b, B27 c, B28a, B28b, B28c)

I BuffDualRailSubtractor (N, NUB, DPa, DPb, DPc, DMa, DMb, DMc, YPa, YPb, YPc, YMa, YMb, YMc) )

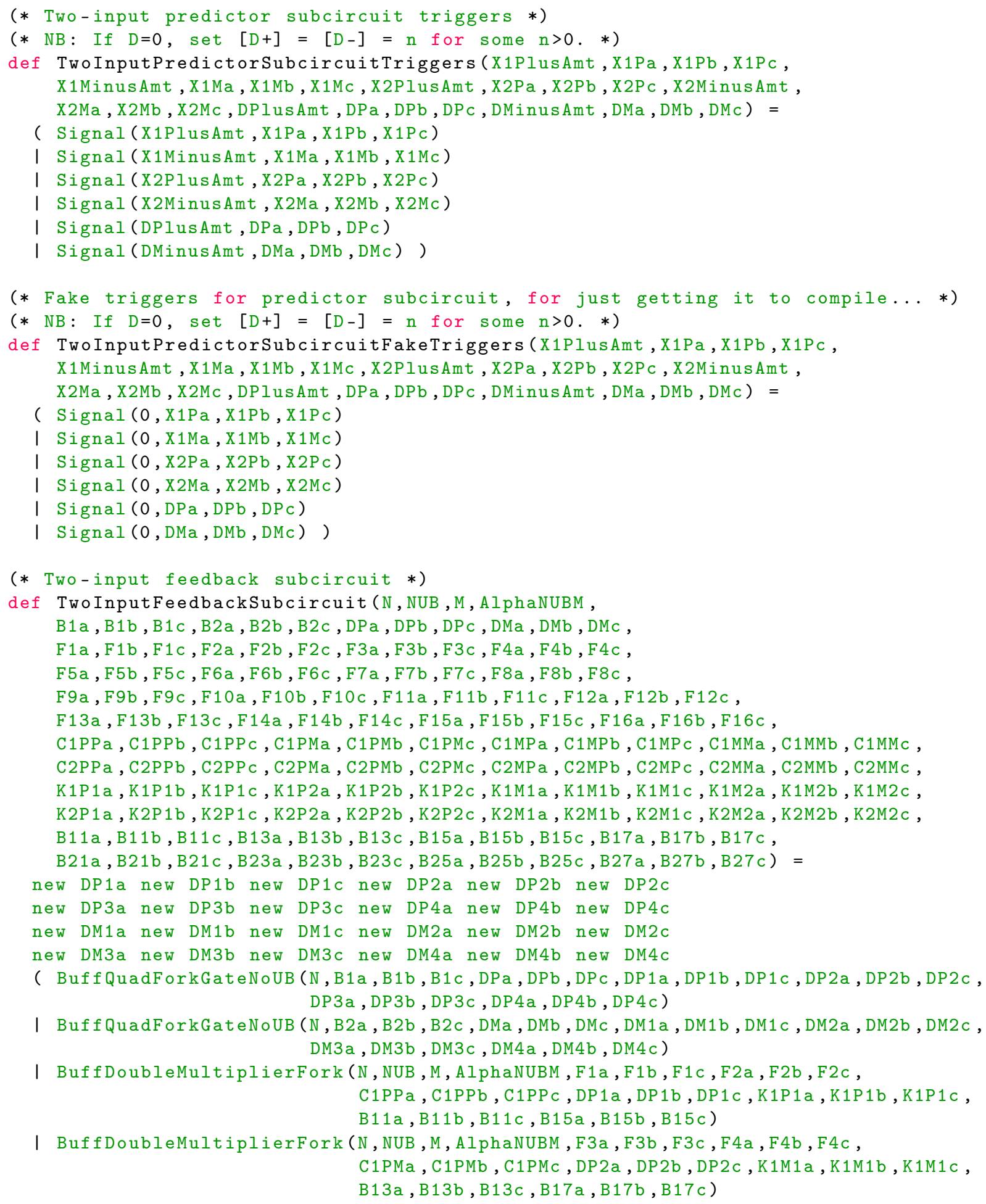


I BuffDoubleMultiplierFork(N, NUB, M, AlphanUBM, F5a, F5b, F5c, F6a, F6b, F6c, C1MPa, C1MPb , C1MPc, DM1a, DM1b, DM1c, K1P2a, K1P2b, K1P2c, $\mathrm{B} 13 \mathrm{a}, \mathrm{B} 13 \mathrm{~b}, \mathrm{~B} 13 \mathrm{c}, \mathrm{B} 17 \mathrm{a}, \mathrm{B} 17 \mathrm{~b}, \mathrm{~B} 17 \mathrm{c})$

I BuffDoubleMultiplierFork(N, NUB, M, AlphanUBM, F7a,F7b, F7c, F8a, F8b , F8c , C1MMa , C1MMb , C1MMc , DM2a , DM2b , DM2c, K1M2a , K1M2b , K1M2c , B11a, B11b, B11c,B15a,B15b, B15c)

I BuffDoubleMultiplierFork(N, NUB, M, AlphanUBM , F9a, F9b, F9c , F10a, F10b, F10c, $\mathrm{C} 2 \mathrm{PPa}, \mathrm{C} 2 \mathrm{PPb}, \mathrm{C} 2 \mathrm{PPC}, \mathrm{DP} 3 \mathrm{a}, \mathrm{DP} 3 \mathrm{~b}, \mathrm{DP} 3 \mathrm{c}, \mathrm{K} 2 \mathrm{P} 1 \mathrm{a}, \mathrm{K} 2 \mathrm{P} 1 \mathrm{~b}, \mathrm{~K} 2 \mathrm{P} 1 \mathrm{c}$, B21a, B21b, B21 c, B25a, B25b, B25c)

I BuffDoubleMultiplierFork(N,NUB, M, AlphanUBM, F11a, F11b,F11c,F12a, F12b, F12c, $\mathrm{C} 2 \mathrm{PMa}, \mathrm{C} 2 \mathrm{PMb}, \mathrm{C} 2 \mathrm{PMC}, \mathrm{DP} 4 \mathrm{a}, \mathrm{DP} 4 \mathrm{~b}, \mathrm{DP} 4 \mathrm{c}, \mathrm{K} 2 \mathrm{M} 1 \mathrm{a}, \mathrm{K} 2 \mathrm{M} 1 \mathrm{~b}, \mathrm{~K} 2 \mathrm{M} 1 \mathrm{c}$, B23a, B23b, B23c, B27a, B27b, B27c)

I BuffDoubleMultiplierFork(N, NUB, M, AlphanUBM, F13a, F13b, F13c, F14a, F14b, F14c, C2MPa, C2MPb , C2MPc, DM3a, DM3b, DM $3 \mathrm{c}, \mathrm{K} 2 \mathrm{P} 2 \mathrm{a}, \mathrm{K} 2 \mathrm{P} 2 \mathrm{~b}, \mathrm{~K} 2 \mathrm{P} 2 \mathrm{c}$, B23a, B23b, B23c, B27a, B27b, B27c)

I BuffDoubleMultiplierFork(N, NUB, M, AlphanUBM, F15a,F15b, F15c,F16a, F16b, F16c, C2MMa , C2MMb , C2MMc , DM4a, DM4b , DM4c, K2M2a, K2M2b, K2M2c , $\mathrm{B} 21 \mathrm{a}, \mathrm{B} 21 \mathrm{~b}, \mathrm{~B} 21 \mathrm{c}, \mathrm{B} 25 \mathrm{a}, \mathrm{B} 25 \mathrm{~b}, \mathrm{~B} 25 \mathrm{c})$ )

(* Two-input feedback subcircuit triggers *)

def TwoInputFeedbackSubcircuitTriggers (NUB, B1a, B1b, B1c, B2a, B2b, B2c,

$\mathrm{C} 1 \mathrm{PPa}, \mathrm{C} 1 \mathrm{PPb}, \mathrm{C} 1 \mathrm{PPc}, \mathrm{C} 1 \mathrm{PMa}, \mathrm{C} 1 \mathrm{PMb}, \mathrm{C} 1 \mathrm{PMc}, \mathrm{C} 1 \mathrm{MPa}, \mathrm{C} 1 \mathrm{MPb}, \mathrm{C} 1 \mathrm{MPc}, \mathrm{C} 1 \mathrm{MMa}, \mathrm{C} 1 \mathrm{MMb}, \mathrm{C} 1 \mathrm{MMc}$, $\mathrm{C} 2 \mathrm{PPa}, \mathrm{C} 2 \mathrm{PPb}, \mathrm{C} 2 \mathrm{PPc}, \mathrm{C} 2 \mathrm{PMa}, \mathrm{C} 2 \mathrm{PMb}, \mathrm{C} 2 \mathrm{PMc}, \mathrm{C} 2 \mathrm{MPa}, \mathrm{C} 2 \mathrm{MPb}, \mathrm{C} 2 \mathrm{MPc}, \mathrm{C} 2 \mathrm{MMa}, \mathrm{C} 2 \mathrm{MMb}, \mathrm{C} 2 \mathrm{MMc})=$

( Signal (NUB, B1a, B1b, B1c)

I Signal(NUB, B2a,B2b,B2c)

I Signal (1, C1PPa, C1PPb, C1PPc)

I Signal (1, C1PMa, C1PMb, C1PMc)

I Signal (1, C1MPa, C1MPb, C1MPc)

I Signal (1, C1MMa, C1MMb, C1MMc)

I $\operatorname{Signal}(1, \mathrm{C} 2 \mathrm{PPa}, \mathrm{C} 2 \mathrm{PPb}, \mathrm{C} 2 \mathrm{PPC})$

I Signal (1, C2PMa, C2PMb, C2PMc)

I Signal (1, C2MPa, C2MPb, C2MPc)

(* Unbuffer the gate that copies $\mathrm{D}+*$ )

(* Unbuffer the gate that copies D- *)

(* Constant input for input $1+/+$ feedback $*$ )

(* Constant input for input $1+/-$ feedback $*$ )

(* Constant input for input $1-/+$ feedback *)

(* Constant input for input $1-/-$ feedback *)

(* Constant input for input $2+/+$ feedback $*$ )

(* Constant input for input $2+/-$ feedback $*$ )

(* Constant input for input $2-/+$ feedback $*$ )

I Signal (1, C2MMa, C2MMb, C2MMc) ) (* Constant input for input 2 -/-feedback *)

(* Fake triggers for feedback subcircuit, for just getting it to compile... *)

def TwoInputFeedbackSubcircuitFakeTriggers (NUB, B1a, B1b, B1c, B2a, B2b, B2c, $\mathrm{C} 1 \mathrm{PPa}, \mathrm{C} 1 \mathrm{PPb}, \mathrm{C} 1 \mathrm{PPc}, \mathrm{C} 1 \mathrm{PMa}, \mathrm{C} 1 \mathrm{PMb}, \mathrm{C} 1 \mathrm{PMc}, \mathrm{C} 1 \mathrm{MPa}, \mathrm{C} 1 \mathrm{MPb}, \mathrm{C} 1 \mathrm{MPc}, \mathrm{C} 1 \mathrm{MMa}, \mathrm{C} 1 \mathrm{MMb}, \mathrm{C} 1 \mathrm{MMc}$, $\mathrm{C} 2 \mathrm{PPa}, \mathrm{C} 2 \mathrm{PPb}, \mathrm{C} 2 \mathrm{PPc}, \mathrm{C} 2 \mathrm{PMa}, \mathrm{C} 2 \mathrm{PMb}, \mathrm{C} 2 \mathrm{PMc}, \mathrm{C} 2 \mathrm{MPa}, \mathrm{C} 2 \mathrm{MPb}, \mathrm{C} 2 \mathrm{MPc}, \mathrm{C} 2 \mathrm{MMa}, \mathrm{C} 2 \mathrm{MMb}, \mathrm{C} 2 \mathrm{MMc})=$

( Signal $(0, B 1 a, B 1 b, B 1 C) \quad(*$ Unbuffer the gate that copies D+ $*$ )

I Signal (0,B2a,B2b,B2c) (* Unbuffer the gate that copies D- *)

I Signal (0,C1PPa, C1PPb,C1PPc) (* Constant input for input $1+/+$ feedback *)

I Signal (0,C1PMa, C1PMb,C1PMc) (* Constant input for input $1+/-$ feedback *)

I Signal (0,C1MPa, C1MPb,C1MPc) (* Constant input for input $1-/+$ feedback *)

I Signal (0,C1MMa, C1MMb, C1MMc) (* Constant input for input $1-/-$ feedback *)

I Signal (0, C2PPa, C2PPb, C2PPc) (* Constant input for input $2+/+$ feedback *)

I Signal (0,C2PMa, C2PMb, C2PMC) (* Constant input for input $2+/-$ feedback *)

I Signal (0, C2MPa, C2MPb , C2MPc) (* Constant input for input $2-/+$ feedback *)

I Signal (0, C2MMa, C2MMb, C2MMc) ) (* Constant input for input 2 -/- feedback *)

(* For the two-input predictor subcircuit *)

$(* N B$ : If $D=0$, set $[D+]=[D-]=n$ for some $n>0$.* $)$

def Predictor $\mathrm{N}=100000$

def PredictorNUB $=100$

def PredictorW1Plus $=2$

def PredictorW1Minus $=5$

def PredictorX1PlusAmt $=0$

def PredictorX1MinusAmt $=5$

def PredictorW2Plus $=4$

def PredictorW2Minus $=1$ 


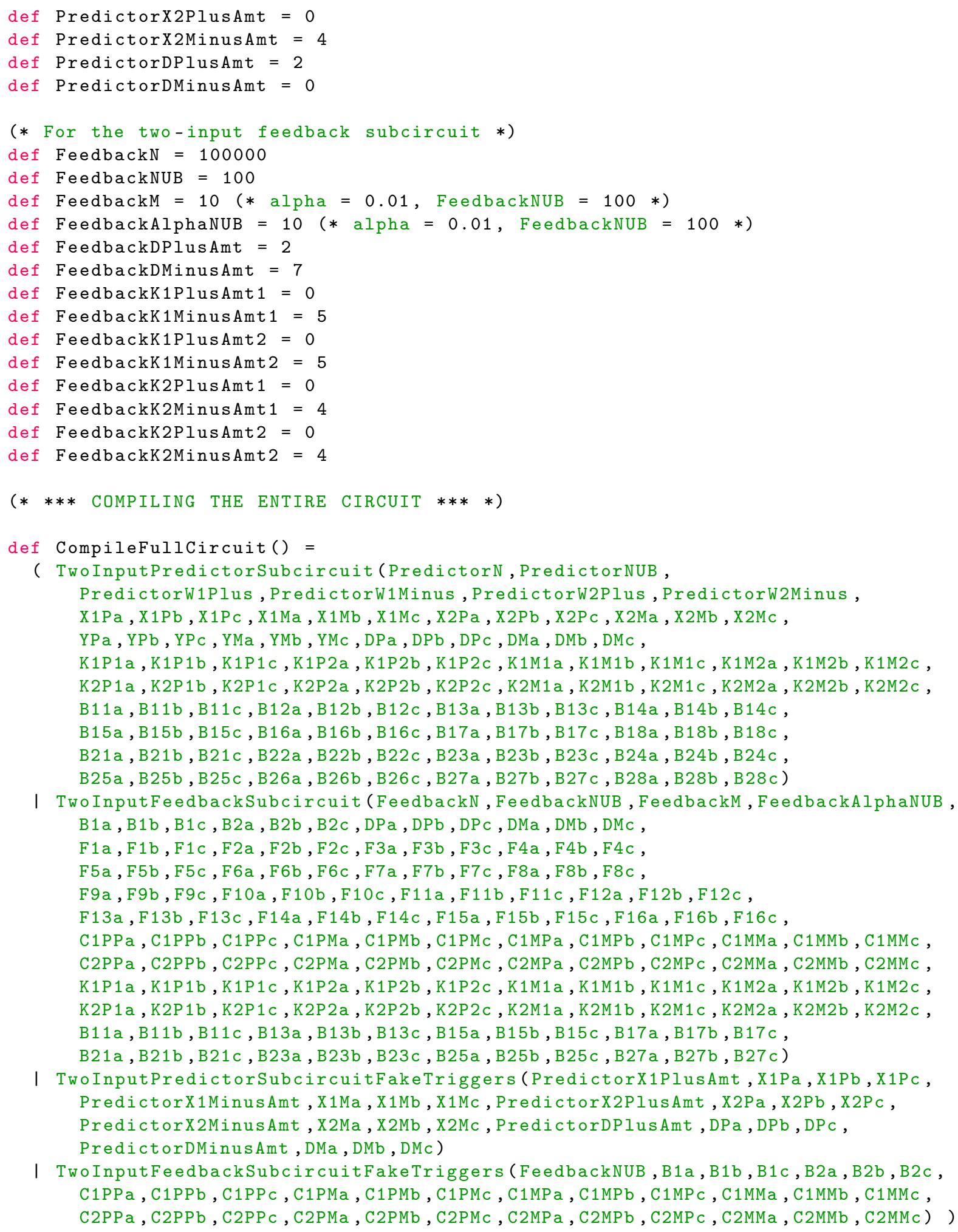

I TwoInputFeedbackSubcircuit (FeedbackN, FeedbackNUB, FeedbackM, FeedbackAlphaNUB, $\mathrm{B} 1 \mathrm{a}, \mathrm{B} 1 \mathrm{~b}, \mathrm{~B} 1 \mathrm{c}, \mathrm{B} 2 \mathrm{a}, \mathrm{B} 2 \mathrm{~b}, \mathrm{~B} 2 \mathrm{c}, \mathrm{DPa}, \mathrm{DPb}, \mathrm{DP} \mathrm{c}, \mathrm{DMa}, \mathrm{DMb}, \mathrm{DMc}$, 


\section{S5 Reference Implementation of Learning Algorithm}

In addition to ODE simulations of our DNA learning circuit model, we also carried out the identical learning procedure using a reference implementation of the stochastic gradient algorithm programmed directly in Python. This allowed us to compare the results from the simulated DNA circuit directly with the results from the reference implementation. The Python function that implements the reference implementation is presented below, and includes the option to specify noise terms (X1Noise, $\mathrm{X} 2$ Noise and DNoise) to superimpose on each training instance value (X1, X2 and D).

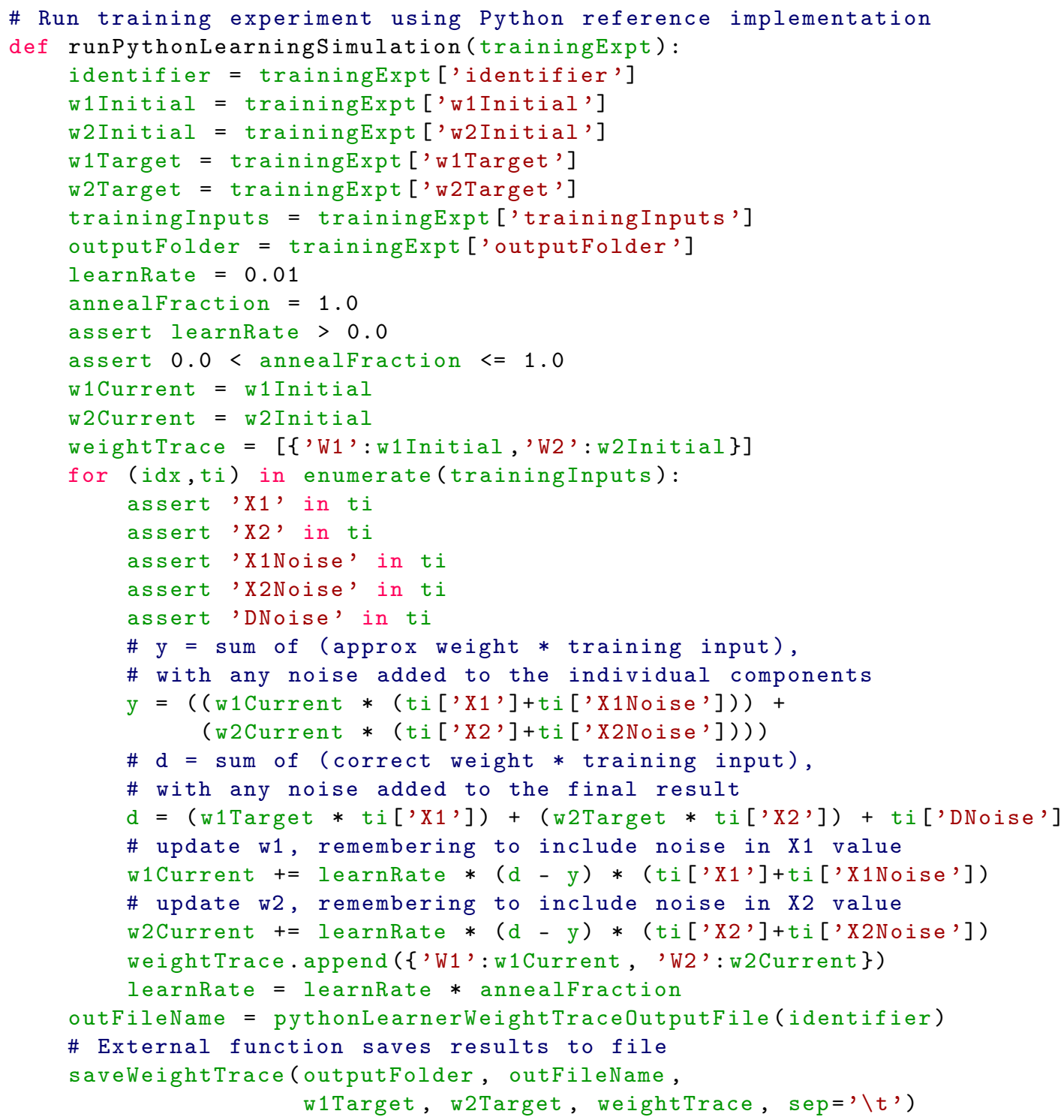

\section{References}

[1] Lakin MR, Youssef S, Polo F, Emmott S, Phillips A. Visual DSD: a design and analysis tool for DNA strand displacement systems. Bioinformatics. 2011;27(22):3211-3213.

[2] Lakin MR, Youssef S, Cardelli L, Phillips A. Abstractions for DNA circuit design. J R Soc, Interface. 2012;9(68):470486. 